\title{
Handbook for gas filled rf cavity aficionados'
}

\author{
A.V. Tollestrup, Moses Chung, Katsuya Yonehara \\ Version 1.01 4-29-2009 \\ Fermilab TM-2430-APC
}

\subsection{Introduction}

The use of hydrogen gas filled RF cavities in muon cooling channels has been proposed by Rolland Johnson [1]. Impressive results have been obtained toward attaining high voltage gradients and rapid training in preliminary tests done at the FNAL MTA facility. However, so far it has not been possible to test them under conditions where they were subject to the transversal of a high intensity particle beam. This note is an attempt to bring together a description of some of the pertinent physical processes that take place in the dilute plasma that is generated in the hydrogen gas by the beam. Two effects dominate. The first is that the free electrons generated can load down the cavity and transfer its energy to heating the gas. The second is a question of what happens to the plasma in the longer term. There is an enormous literature on the subject of the subject of dilute hydrogen plasmas and we can tap into this information in order to understand and predict the behavior of the cavity.

Another very interesting subject is the behavior of the limiting breakdown voltage of the cavity vs. the gas density. This is a subject that has an extensive literature in the case of vacuum RF cavities and is a subject that is still note well understood. Since in general RF cavities are pushed to their limit, it is important to understand what governs the limiting voltage. The gas filled cavity opens up a new way to study this limit and this note will explore the pertinent physical processes.

This note is an attempt to bring together in one spot a discussion of the phenomenon and furnish a consistent framework for understanding and predicting the behavior of gas filled RF cavities. It will be updated as we learn more. There is also a set of Mathematica Notebooks that are available for the asking that will carry out some of the more complicated calculations and are consistent with the present literature. The notebooks should make a common platform for performing calculations as the pertinent equations are clearly displayed where they can be critiqued and corrected, if necessary. To this end it has been necessary to search the literature for the behavior of electrons in gasses and in particular hydrogen. These appear in the references, but one of the authors (AVT) has these and many more that can be made available by request.

There is also a section on what we don't know and what we would like to know. These questions are based on the work in this note and are intended to help in the direction of the upcoming experiments at MTA. 


\subsection{Some Experimental Data}

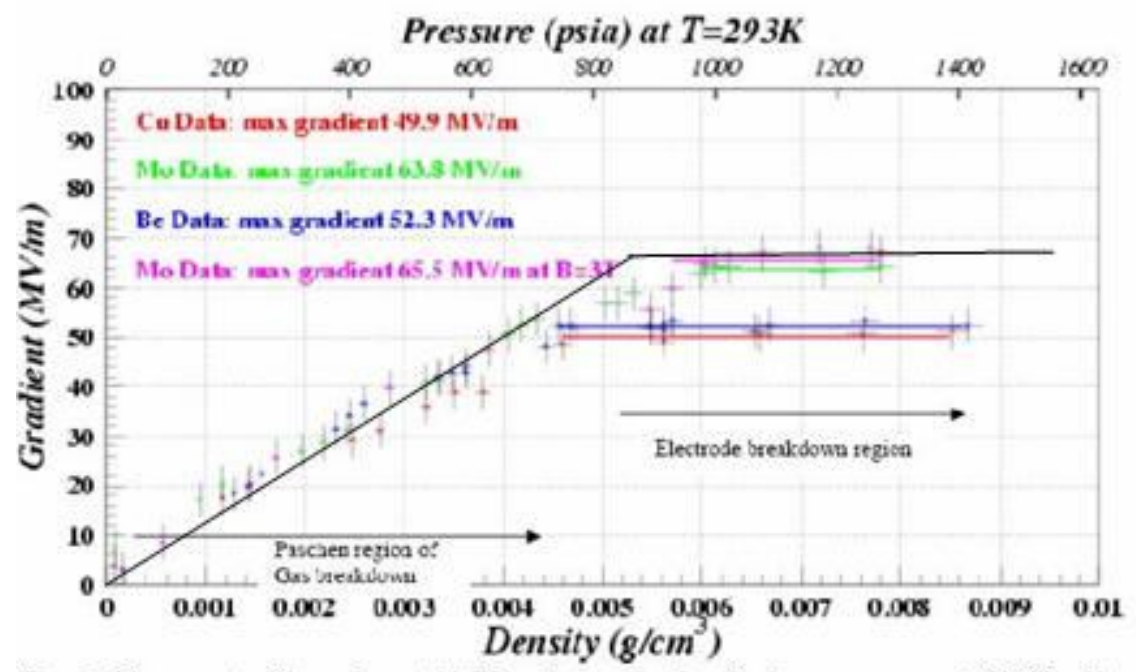

Tigare 3: Meastrecueats of the naxincuas stable TC gradient as a fuoctiva of tydiogea gas pressure at $300 \mathrm{MIII}$ with 50 nagaetic field for turee differeat elesrode materials, copper (red), asolybdeanas (greeg), asd berylliana (bive). As the

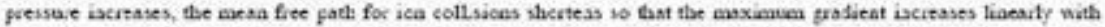
fresjexe. At sufficienly high pretsuec, the martienn gadent is determined by electrode breakilown and hes litile if

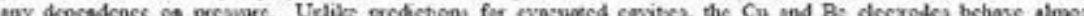

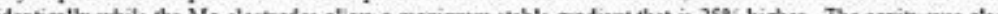
identically while the

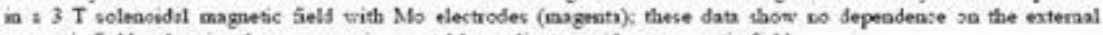
enagentic fielt, achering the came maximam ctahls gadient as wifh mo nagietic field

Fig. 2.1 The linearly rising part is the Paschen Region and the flat region is determined by the electrode properties. Ref[1].

The possibility of obtaining very high gradients is very attractive for muon cooling channels. The curve above indicates that there are two regions of interest. The first is the linear slope of break down voltage vs. pressure, called the Paschen region, and the second is the flat region where the pressure variation is flat, but the value depends on the electrode material. The above data was taken in a cavity with no beam and the purpose of this note is to develop a theory of behavior of a gas filled cavity with an intense beam traversing it near the peak of the rf voltage.

\subsection{Physics of the Paschen Region}

Let's first consider the linearly rising part of the curve in Fig.2.1. The variable used is the gas density. However in 1889 F. Paschen published a paper showing that a more general variable was $\mathrm{E} / \mathrm{P}$ :

Eq.2.1 $\quad \mathrm{V}_{\text {breakdown }}=f(E / p)$ 
Historically the units have been volts/cm and Torr. The reason for this dependence is easily seen. If we consider decreasing the distance between molecules by $1 / 2$ and increasing the field gradient $\mathrm{E}$ by two, the physics processes should all be the same as a free electron will gain the same amount of energy between collisions. It is also seen from this argument that the gas density should be a better variable than pressure and so one will find E/n used in the literature with a unit which is called the "Td" where

$$
\text { Eq.2.2 } 1 \mathrm{Td}=1 \text { volt } / \mathrm{cm} / 10^{17} \text { molecules } / \mathrm{cm}^{3} \text {. }
$$

The function $f$ is linear in the region of interest here, but at lower pressures it goes thru a minimum called the "Paschen Minimum" and then rises as the pressure is lowered and the mean free path starts to approach the dimension of the vessel. We will confine ourselves to the high pressure side of this curve.

The breakdown occurs when a free electron can gain enough energy to ionize the gas and thus generate more electrons which continue to form a Townsend Avalanche. This is characterized by an exponential growth in the number of ions with distance:

$$
\text { Eq.2.3 } n(x)=n(0) \operatorname{Exp}(\alpha x)
$$

and $\alpha$ is called the First Townsend Coefficient and a measurement of it as a function of E/p is shown in Fig.[2.2] below. Let's put in some numbers. For the linear part of the curve in Fig.2.1, we have

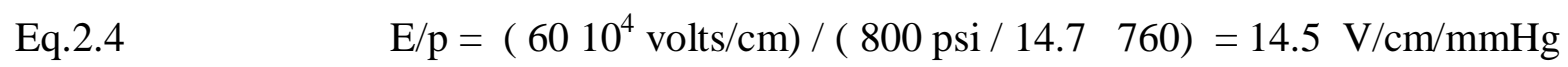

This is the boundary of the breakdown region all along the linearly rising part of the curve. Using $\mathrm{E} / \mathrm{P}=14.5$, Figure 2.2 shows that $\alpha / \mathrm{p}$ is small, perhaps .001 . Note that it will go to zero when an electron cannot gain the 15.5 volts of energy needed to ionize the hydrogen molecule. Using the value of $\alpha / p=.001$, we can calculate $\alpha$ for a pressure of $800 \mathrm{psi}$, which is on the corner of the Paschen region and find :

$$
\text { Eq.25 } \alpha=.001 \mathrm{p}(\text { Torr })=.001(800 / 14.7) 760=41.4 \mathrm{~cm}^{-1}
$$

This will scale with pressure along the linear region of the curve. However, Eq.2.3 does not predict breakdown as it just gives the gas amplification at any given $\mathrm{E} / \mathrm{p}$ and for the case we just considered the ionization doubles every $1 / 41.1 \mathrm{~cm}=0.24 \mathrm{~mm}$. So although the amplification may be very large, the equation does not predict breakdown. For breakdown to occur there needs to be an additional feedback mechanism that causes instability and makes the current increase without bound. This leads to a second equation for the current in a gap:

Eq.2.6 $n(x)=n(0) \operatorname{Exp}(\alpha x) /[1-\gamma(\operatorname{Exp}(\alpha x)-1]]$

Where $\gamma$ is the second Townsend Coefficient and a singularity in the denominator is clearly possible. There are different sources driving $\gamma$ growth. For instance, in the early measurements it 
was found that photons emitted by recombination of the electrons could hit the cathode and produce photo electrons which augmented the current. It is also possible that positive ions hitting the cathode can eject additional electrons, thus increasing the gap current and providing the feedback for breakdown. In any case since the term multiplying $\gamma$ can be very large, and as a result a very small $\gamma$ can produce a zero in the denominator.
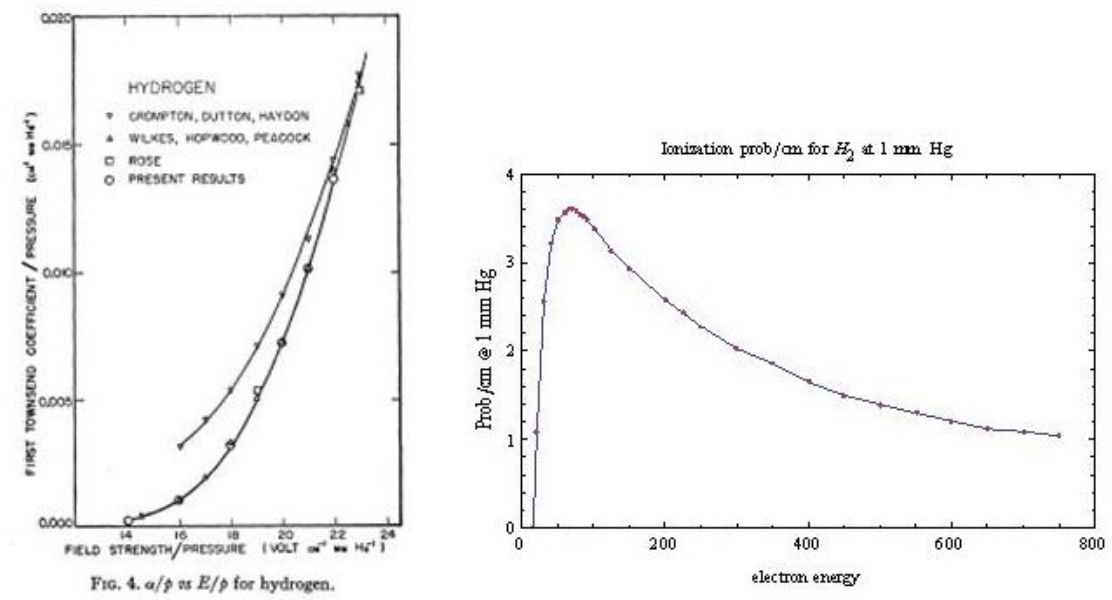

Fig. 2.2. X (left) from [2] and the ionization cross section for electrons striking a hydrogen molecule on the right[3]. The threshold for ionization of the molecule is $15.5 \mathrm{eV}$.

The motion of an electron in a dense gas is a stochastic process and so it is customary to consider the behavior of a "swarm" of electrons and calculate or measure the various distribution functions that characterize the ensemble. If each collision of the electron with a molecule was completely elastic, then after the electron diffused a distance in the $\mathrm{z}$ direction equal to $15.5 \mathrm{eV} / \mathrm{E}$ it would have enough energy to ionize a molecule. For E equal $60 \mathrm{MV} / \mathrm{m}$ this would be about $0.2510^{-4} \mathrm{~cm}$. But we saw above that the $1 / \mathrm{e}$ distance was about $1 / 40 \mathrm{~cm}$.

However, the collisions are not completely lossless. For instance the electron looses roughly $2 \mathrm{~m}_{\mathrm{e}} /$ $M$ of its energy due to the recoil off of $\mathrm{M}$ in completely elastic collisions. This is the case with $\mathrm{He}$ which is monatomic with no low lying states that can be excited. Its breakdown field is very low because the energy loss per collision is very small and it is easy for an electron to gain enough energy to cause ionization. However, the $\mathrm{H}_{2}$ molecule has many low lying vibrational and rotational states in the .01 to $0.1 \mathrm{eV}$ region. The result is that the electron comes into an equilibrium distribution with a rather low mean energy. It is the tail of this distribution that reaches out to 15.5 $\mathrm{eV}$ and causes the avalanche to start. If the velocity distribution doesn't reach out to $15.5 \mathrm{eV}$, no avalanche will form and the swarm will just drift with a mean velocity. This is the case for an electron below the E/p = 14.5 line in Fig.2.1. Once this line is crossed, the avalanche process starts. This is nicely demonstrated by some simulations reported by D. V. Rose of Voss Scientific and shown in the LE Muon Collider Workshop at FNAL April 21-25, 2008. Fig.2.3 below shows the results for the plasma density growth for a swarm in $\mathrm{H}_{2}$ at a density of $.002 \mathrm{~g} / \mathrm{cm}^{3}$ and fields of 10 , 25, and $50 \mathrm{MV} / \mathrm{m}$ which are below avalanche level, at the Paschen Limit line, and well above this limit. 


\section{OD simulations of RF breakdown are in agreement with experiments in $\mathrm{H}_{2}$ at $0.002 \mathrm{~g} / \mathrm{cm}^{3}$ :}

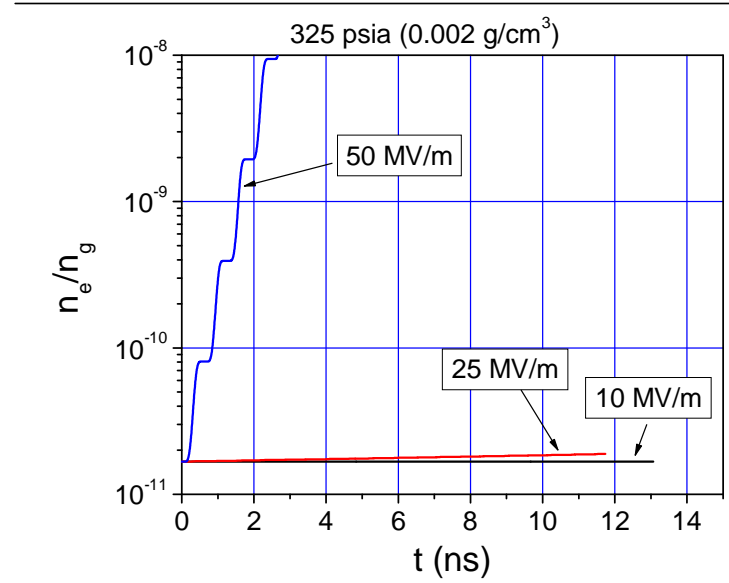

Seed plasma population has a density of $10^{10} \mathrm{~cm}^{-3}$, a very small fraction of the initial neutral gas density $\left(6 \times 10^{20} \mathrm{~cm}^{-3}\right)$.

The $25 \mathrm{MV} / \mathrm{m}$ simulation shows a very slow growth in electron density (red curve) and the $50 \mathrm{MV}$ simulation (blue curve) shows an extremely rapid breakdown of the gas.

At $25 \mathrm{MV} / \mathrm{m}$, breakdown is initially slow but finite (borderline Paschen level).

$50 \mathrm{MV} / \mathrm{m}$ is well above Paschen level.

Fig.2.3 Data from a simulation of D. V. Ross. See Ref[4]

A short discussion of the swarm dynamics is in order. An electron in $\mathrm{H}_{2}$ and an electric field $\mathrm{E}$ will make a random path thru the gas but will have an average drift in the direction of E. Since this is a stochastic process, it will gain energy from the field and loose it to the gas thru inelastic collisions and will arrive at some equilibrium distribution. If there is no electric field, this distribution will be a Gaussian with energy determined by the ambient gas temperature. However, once a field is applied the electron swarm absorbs energy from the field and comes to some new distribution. This distribution is dependent on the properties of the gas and the characteristics of the inelastic cross section variation with velocity. This is a complicated subject, but fortunately there are only two features that we need. The first was mentioned above, $\alpha / \mathrm{p}$ and is shown in Fig.2.2 At a given E/P it gives us the distance that an electron must diffuse before it has enough energy to ionize a molecule. The second information that we need is the average velocity of the swarm in the direction of the field as a function of $\mathrm{E}$ and $\mathrm{P}$ and this in encoded in a parameter $\mu$ called the mobility.
Eq.2.6
$\mathbf{v}=\mu \mathrm{E}$

We can make a simplified derivation of this equation. Mobility is measured by observing the drift velocity of swarm electrons in a field $\mathrm{E}$ and is normalized to the value observed for a gas pressure of 1 Torr. To see the physics, consider an electron moving under the effect of $\mathrm{E}$ and making random collisions with the molecules and changing direction. It has a coherent velocity, $\mathrm{v}$, in the direction of the field and a random motion that is characteristic of the swarm. The temperature of the swarm can be well above the gas temperature as explained above because of the absorption of energy from the field. However it will have a coherent drift given by:

$$
\mathrm{v}=1 / 2 \text { at }=1 / 2 \mathrm{e} / \mathrm{m} \mathrm{E} \mathrm{t}=1 / 2 \mathrm{e} / \mathrm{m} \mathrm{E}\left\langle\lambda / \mathrm{V}_{\mathrm{r}}\right\rangle
$$


We start with an electron whose random velocity is $\mathrm{V}_{\mathrm{r}}$. It accelerates under the action of the field in the $\mathrm{z}$ direction until it hits a molecule and is deflected its average change of velocity in the $\mathrm{z}$ direction is given by the equation above. The distance it travels is $\lambda$ and its velocity is $\mathrm{V}_{\mathrm{r}}$, the random velocity of the swarm. The distance it travels is given by the total cross section and the gas density:
Eq.2.8
$\lambda=1 / \mathrm{N} \sigma$

Combining with Eq.2.7 gives

Eq.2.9

$$
\mathrm{v}=1 / 2 \mathrm{e} / \mathrm{m}<1 /\left(\mathrm{N} \sigma \mathrm{V}_{\mathrm{r}}\right)>\mathrm{E}=\mu \mathrm{E}
$$

Notice that $\mu$ is a function of $\mathrm{E} / \mathrm{P}$ since if we double $\mathrm{P}$ and halve the distance between molecules then double $\mathrm{E}$ the physics should remain the same. The mobility has been measured for electrons in hydrogen over a wide range pressure, density and temperature. The following two curves show results that are in the range of interest here which vary from the Paschen Limit at E/P $=14$ to perhaps values as low as $\mathrm{E} / \mathrm{P}=0.1$ for dense gas used in a cooling channel.

In the case of high field, the swarm random velocity of the electrons is much above that of the molecules. The energy from the coherent motion in the field is fed into random motion by scattering. So the electron swarm may have a mean kinetic energy of several eV. The following figures give the available data on mobility of electrons in hydrogen.
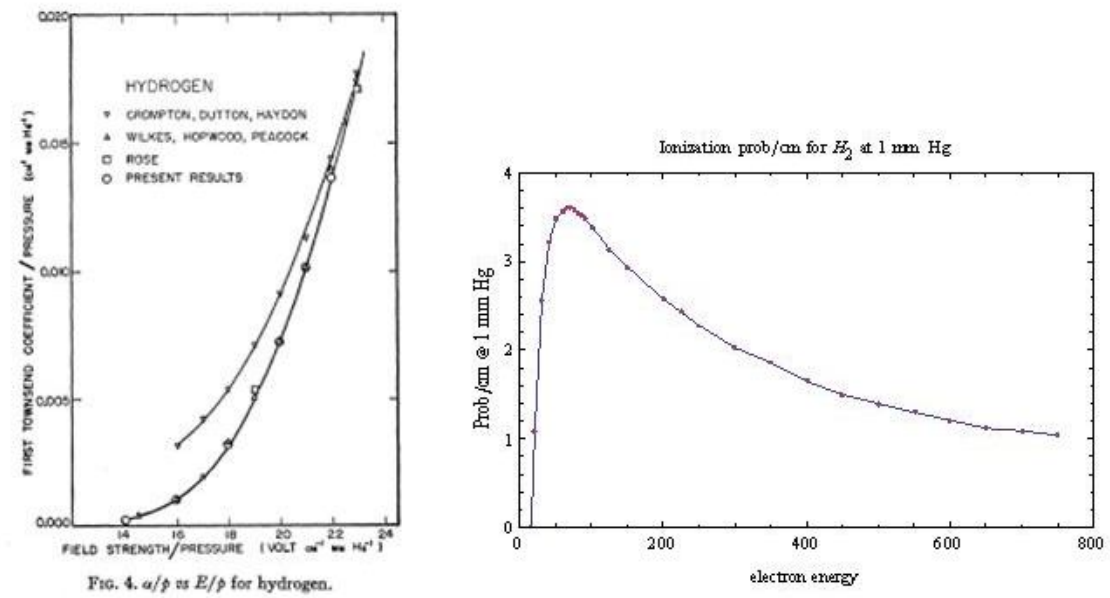

Fig[2.4] The curve on the left is from Pack and Phelps [5], that on the right from Bartels [6]. The region of E/P between 0.5 and 14 is pertinent.

Note that in the range of $\mathrm{E} / \mathrm{P}<0.1$, the drift velocity is not a simple function of only $\mathrm{E} / \mathrm{P}$. However for $0.1<\mathrm{E} / \mathrm{P}<10$ the assumption is good to better than $30 \%$ which will satisfy our needs. Remember that breakdown is at an E/P of 14, but in a cooling channel, the density may be high and the operating point far from breakdown. This could have two effects. The low mobility could reduce the $\mathrm{rf}$ loading by the electrons.... a good effect! The deviations at very low values of E/P are where the swarm energy is approaching the kinetic temperature of the gas molecules which is a region we do not enter as long as there is a high field in the cavity. When the field is removed, the very small 
mobility could impede the removal the residual ionization and decrease the recombination rate..... a bad effect! We will discuss this problem later.

Heylen [7] has fit the data at low density with an empirical equation that is good to $16 \%$ in the region $0.1<\mathrm{E} / \mathrm{P}<100$. He gives two functions one is for the mean random energy in eV for an electron in the swarm and the other is for the mobility. The fits are given below along with the equation to calculate the drift velocity as a function of $\mathrm{E} / \mathrm{P}$ and a plot (Note: there is a misprint in the paper. The following equations are correct). Roughly, the rms $\epsilon_{\mathrm{m}}$ for an AC field should be between the DC value and $1 / 2$ that value and the drift velocity and the internal swarm rms velocity multiplied by 1.0 - 0.7 because the rate that electrons can gain energy from the field is different than the rate that they give up energy thru collisions. If the damping rate is fast enough so that they are always in equilibrium over the RF cycle, then the factor $1 / 2$ applies, otherwise the energy may be higher. Hydrogen with it high inelastic cross section should follow the field.

$$
\epsilon_{\mathrm{m}}=0.357(\mathrm{E} / \mathrm{P})^{0.71}
$$

$$
\mu[\mathrm{E} / \mathrm{P}]=1.7210^{-2}\left[1-2.410^{-2}(\mathrm{E} / \mathrm{P})^{0.71}\right]^{-1.75}(\mathrm{E} / \mathrm{P})^{-.53}
$$

$$
\mathrm{v}[\mathrm{E} / \mathrm{P}]=\mu[\mathrm{E} / \mathrm{P}] \mathrm{E} / \mathrm{P} 5.9310^{7} \mathrm{~cm} / \mathrm{sec} \quad \text { where } \mathrm{E} / \mathrm{P} \text { is in } \mathrm{V} / \mathrm{cm} / \text { torr }
$$

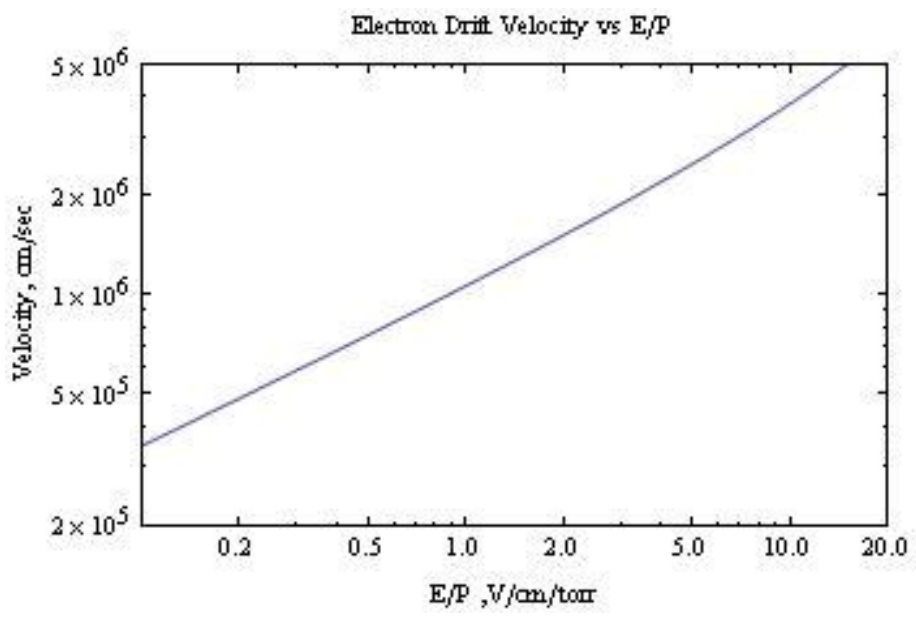

Fig.2.5. Drift velocity vs E/P from Heylens fit. [7].

Using the equation above, we find $\Theta_{\mathrm{m}}=0.357(14)^{0.71}=2.33 \mathrm{eV}$ along the Paschen limit for breakdown. This corresponds to a mean electron velocity in the swarm of $910^{7} \mathrm{~cm} / \mathrm{sec}$, a value 90 times greater than the swarm drift velocity given by Eq.2.10c and shown in Fig.2.5. The calculation of the distribution function for velocity from the measured elastic and inelastic cross section has been attempted with some success. The distribution is not Maxwellian in general but for hydrogen the deviations from this distribution are not large. Suffice it to note that the tail of the distribution must extend to the ionization value for the $\mathrm{H}_{2}$ molecule at $15.37 \mathrm{eV}$.

There is an additional interesting subject that we must address and that has to do with electrons injected into the gas by small sharp points on the electrode surfaces. If we are below $\mathrm{E} / \mathrm{P}=14$, these electrons cannot propagate and cause breakdown but the question of what happens to them is interesting. For instance, are their enough of them to load the cavity? And if we are above E/P=14 
how many RF cycles does it take for the disturbance to propagate across the cavity and cause an arc? Is light emitted? If so we would have a new tool, similar to the dark current emission in vacuum cavities that could be used to study gas filled cavity behavior.

\subsection{The plateau region}

Consider next a point at a pressure of 1000 psia in Fig. 1 which is well into the plateau region. A linear extrapolation of the Paschen's Law to this density would indicate a breakdown field of about $75 \mathrm{MV} / \mathrm{m}$ and yet break down is occurring at about $62 \mathrm{MV} / \mathrm{m}$. The model for this region is dependent on information gleaned from the study of vacuum cavities. The assumption is made that the cavity surface is similar to the surface that has been studied in the vacuum case $[8,9,10]$. In the vacuum case field emission has taught us a lot about the emitters on the cavity surfaces. We have not yet developed the comparable tools for the gas filled case, although if one could observe light from excited gas atoms before the breakdown occurs, we would have a way of extracting prebreakdown information.

We will assume that the metal surface is covered by many small point like projections, called emitters. The field at the tip of thee emitters is great enough so that field emission takes place and injects electrons into the gas. The local field is high enough that these electrons can form an avalanche and increase the local charge density. (Note: If the field is high enough for field emission at the tip of the emitter, it will also be well above the avalanche limiting E/P by even as much as a factor of 10!) The generated charge density and distribution must be such that as it moves away from the emitter it can distort the cavity $\mathrm{E}$ field (which is only $62 \mathrm{MW} / \mathrm{m}$ ) at its leading edge to be equal to or greater than $75 \mathrm{MV} / \mathrm{m}$, the Paschen limit, and thus cause the avalanche to propagate across the cavity. This is a complicated process and needs to be simulated. For instance:

1. The rf field is reversing and some of the electrons can be sucked back into the emitter,

2. The charge density is very high and space charge effects must be important.

3. Positive ions can hit the emitter and cause secondary emission and heating which would be a positive feedback effect.

When the streamer does reach across the cavity, an arc ensues and discharges the cavity in a few cycles (see section[] discussion of the details). In the process, some of the electrode is melted and thrown around which generates more emitters and after many such episodes finally comes into equilibrium and the cavity is said to be "trained". We now take a detour and explore the theory of field emission and some of its consequences:

\subsubsection{Field Emission}

Field emission is governed by the Fowler Nordheim equation [11]:

$$
\begin{aligned}
& j=A \frac{E^{2}}{t(y) \phi} e^{B \phi^{1.5} v[y] / E} \\
& \mathrm{y}=.0362 \mathrm{E}^{1 / 2} / \phi \\
& \mathrm{j}=\text { current density in } \mathrm{A} / \mathrm{cm}^{2} \\
& \mathrm{~A}=154 \quad \mathrm{~B}=6830 \\
& \mathrm{E} \text { is the DC field in } \mathrm{MV} / \mathrm{m}, \quad \phi \text { is the work function in } \mathrm{eV} \\
& \text { The functions } \mathrm{t}(\mathrm{y}) \text { and } \mathrm{v}(\mathrm{y}) \text { are shown below. }
\end{aligned}
$$


This equation is composed of two parts. The first part is the flux of electrons inside the metal that strike the surface and the second is the probability that these electrons can penetrate the barrier at the surface and escape. The figure below shows the potential energy at the surface for an applied field of $1 \mathrm{GV} / \mathrm{m}$.

Potential energy diagram

for $\mathrm{E}=1 \mathrm{GV} / \mathrm{m}$

The horizontal line is at $-4.56 \mathrm{eV}$, the $\phi$ for $\mathrm{W}$

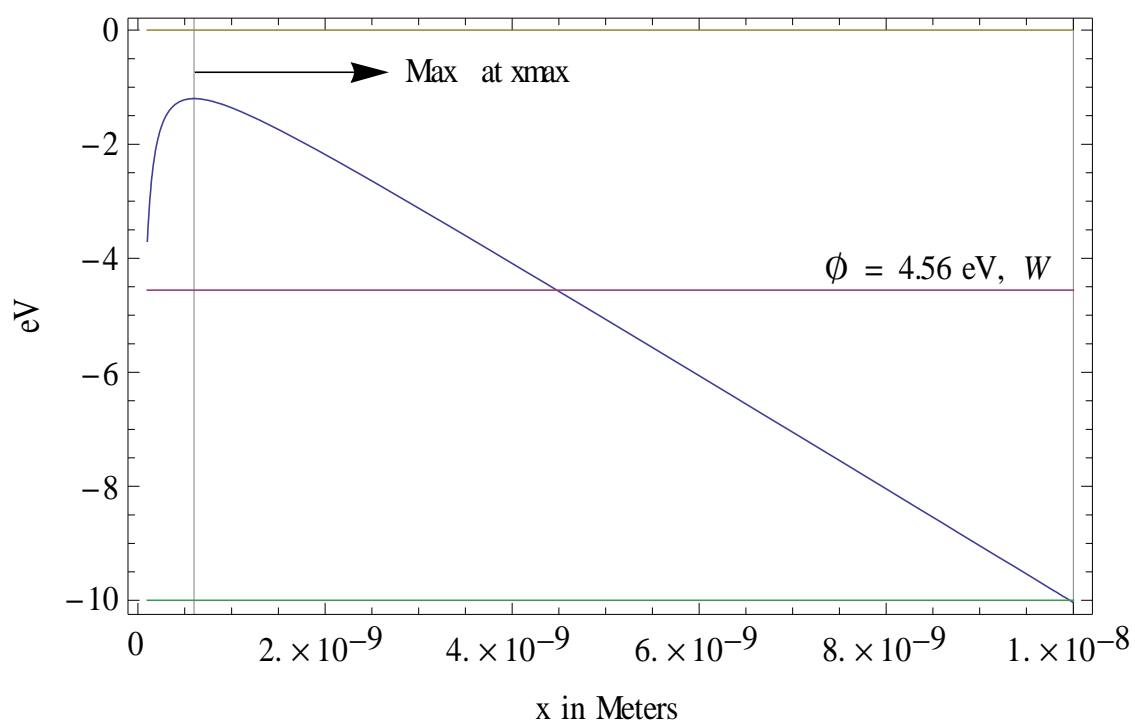

Fig.2.6. The potential energy of an electron outside the surface of tungsten with an applied field of $1 \mathrm{GV} / \mathrm{m}$. The tungsten surface is at the left and the turn over in the curve is caused by the image force attracting the electron toward the surface. The $\mathrm{x}_{\max }=0.610^{-4} \mathrm{E}^{-0.5}$ microns and $\mathrm{V}_{\max }=-1.2 \mathrm{E}^{0.5}$ volts and where $\mathrm{E}$ is in $\mathrm{GV} / \mathrm{m}$. The horizontal line is at 4.56, the work function for tungsten.

The reason the current changes so fast is that as the field is increased, $\mathrm{V}_{\max }$ decreases and the slope of the line increases. Both effects decrease the thickness of the barrier that must be penetrated. Heating the metal will cause some of the electrons to have a higher energy and thus there is a temperature dependence of the current density. This effect only starts to become important at temperatures of around $1000 \mathrm{~K}$ and the correct theory is given in [11]

Quite often authors omit the two functions $t(y)$ and $v(y)$ and in the case of $t(y)$ this is justified as it is close to unity and only modifies the current density by a few percent. However $v(y)$ multiplies the exponent and has a large effect, especially on the size of an emitter and will be included in what follows. The two functions are shown below in Fig.2.7 and have been correctly calculated in 1953 by Burgess and Kroemer [12]. Before this date the expressions used were incorrectly given by Nordheim and were used in many papers. 

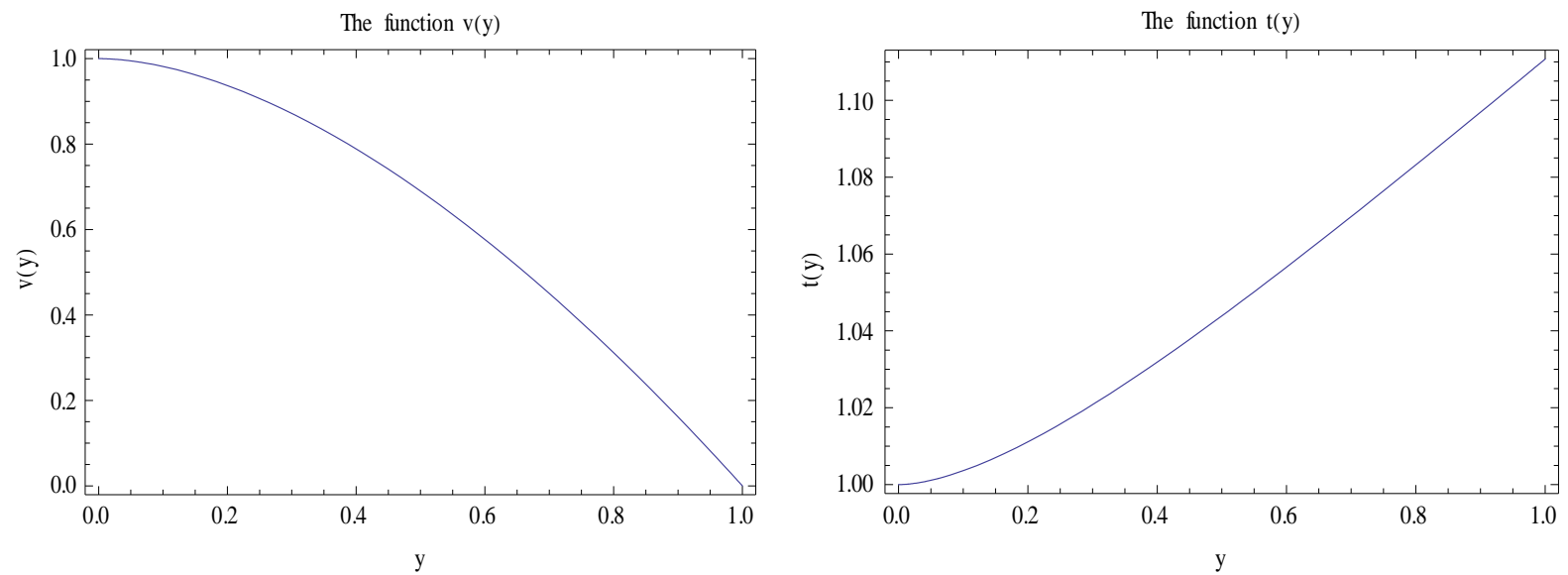

Fig. 2.7 showing the functions $v(y)$ and $t(y)$.

We show below $\mathrm{v}(\mathrm{E})$ for tungsten :

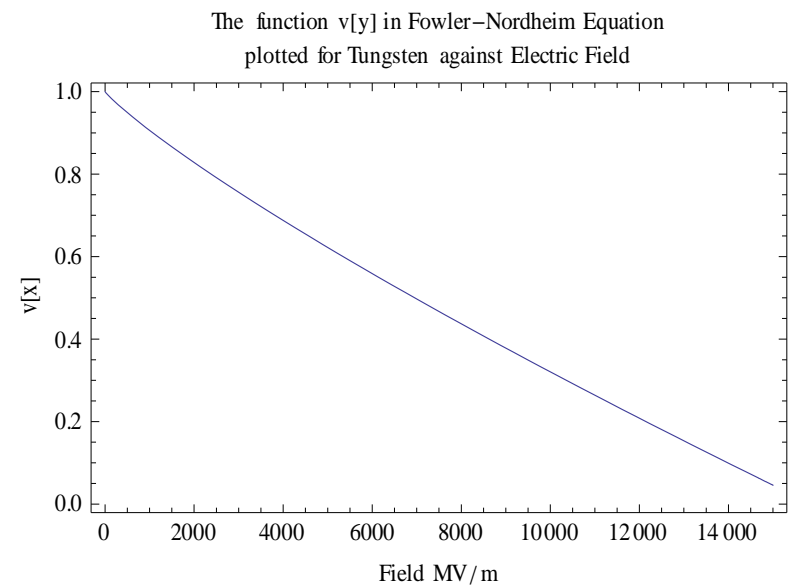

Fig 2.8 Showing the variation of the function $\mathrm{v}$ with applied field for tungsten.

First, the following curve, Fig.2.9, shows the effect of setting $\mathrm{v}$ and $\mathrm{t}$ equal to 1 for a tungsten electrode. Since $v[\mathrm{E}]$ is less than 1 , setting it to 1 in the exponent decreases the emission by a large amount. Also notice that the slope of the curve decreases at as field increases. This provides a direct way of measuring the local field and current density for vacuum cavity where the logarithmic derivative of the dark current can be directly obtained by measuring the secondary $x$-ray intensity as a function of cavity voltage. If in addition, the actual current can be measured it can be combined with the current density to obtain information about the size of the emitters. 
Field Emission Current Density,amps $/ \mathrm{cm}^{2}$

For Tungsten, $\mathrm{wf}=4.56 \mathrm{eV}$

Red set $\mathrm{v}$ and $\mathrm{t}=1$, Green use full expression

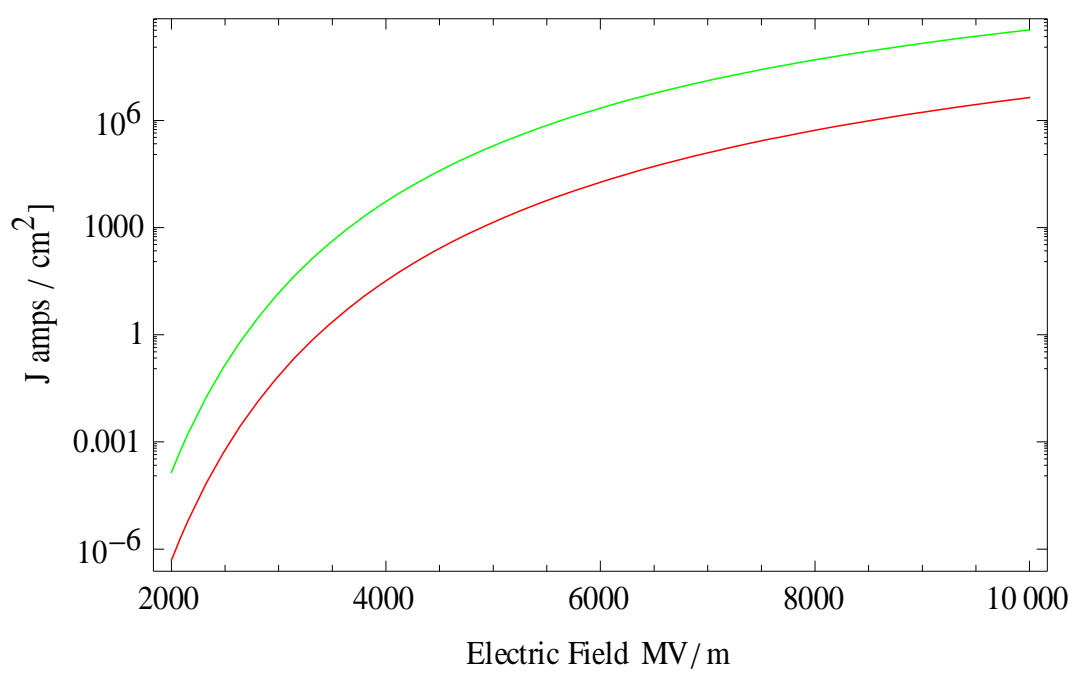

Fig.2.9 Curves for tungsten showing the effect on current density from setting $v(y)=1$.

Next as mentioned, the rf varies and emission occurs only at the peak. The following set of curves on the next page, Fig.2.11 shows the emission for an $800 \mathrm{MHz}$ cavity around the peak of the sine wave. Note the enormous variation of the current density. The Fig.2.10 below summarizes the results by giving the current averaged over a complete RF cycle (emission only on the positive part of the wave form!) and comparing with the corresponding emission from a DC field. The ratio varies due to the variation of the width of the current pulse, being 8.2 at $8 \mathrm{GV} / \mathrm{m}$ and 15 at $2 \mathrm{GV} / \mathrm{m}$.

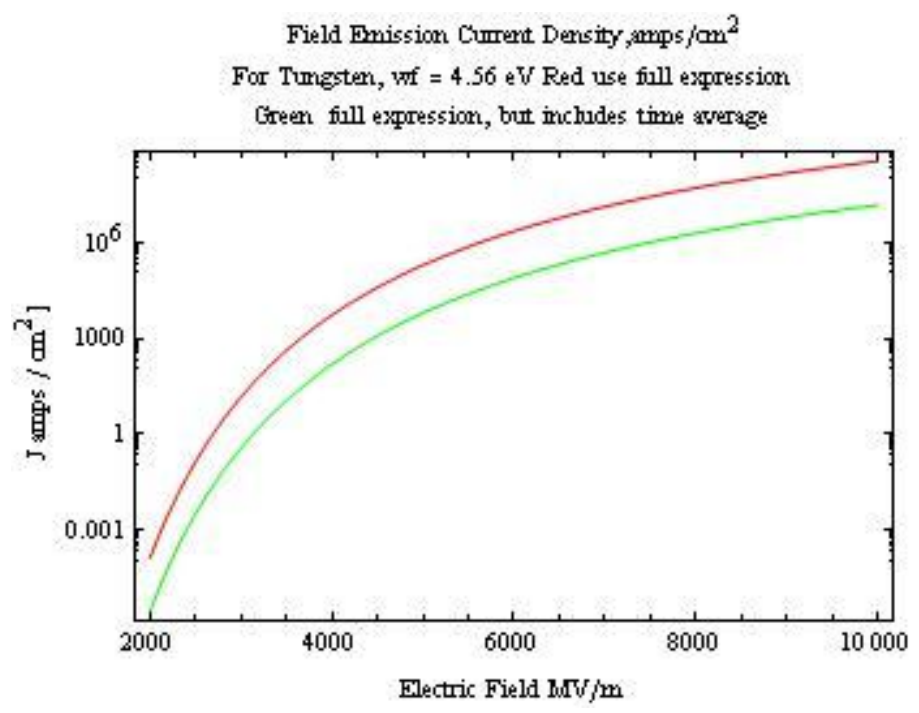

Fig.2.11 This figure compares the field emission from a DC field with that from an RF field. 

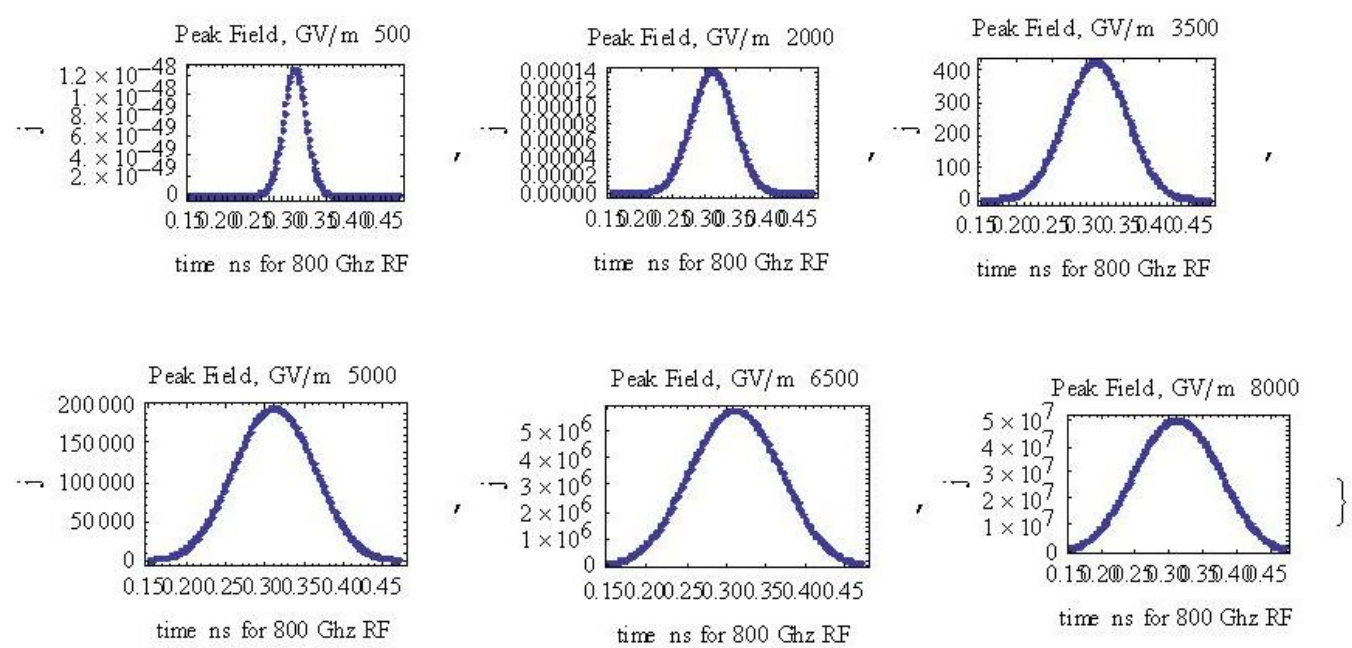

Fig.2.12. Field emission from an emitter in an $800 \mathrm{Mhz}$ cavity around the peak of the rf cycle. The time is in ns and the peak rf field at the emitter is given in GV/m. Note the tremendous variation in $\mathrm{j}$ ! The top curve compares the DC and $\mathrm{AC}$ emission average current vs. field at the asperity.

The next curve, Fig.2.13 compares the current density in an RF field for W, Mo, Cu and Be.

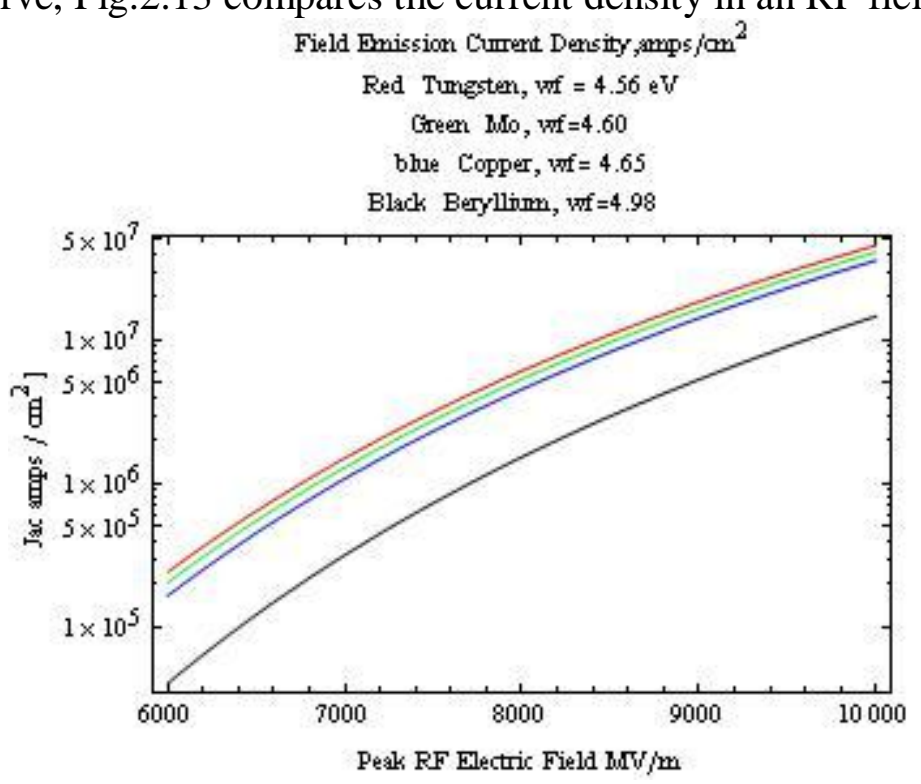

Fig.2.13. The current density averaged over an RF cycle for the four elements $\mathrm{W}, \mathrm{Mo}, \mathrm{Cu}$, and $\mathrm{Be}$ arranged according to increasing work function.

Next, we calculate the logarithmic derivative as a function of E. As mentioned previously, this can be directly measured in a vacuum cavity, but has only been indirectly inferred for the gas filled cavity [13]. The approach used involved measuring the number density of arc pits on the spherical shaped electrodes of the $800 \mathrm{Mhz}$ gas filled cavity and assuming that the pit density variation was due to field emission, an untested assumption. The data is shown in Fig.2.14. 


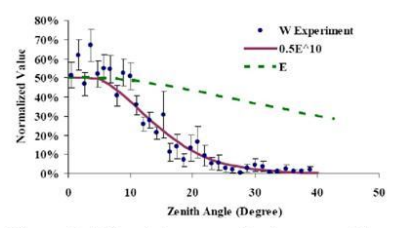

Figure 12: $\mathrm{W}$ breakdown area fraction vs. zenith angle.

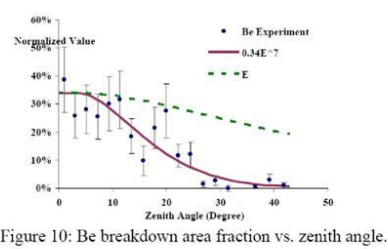

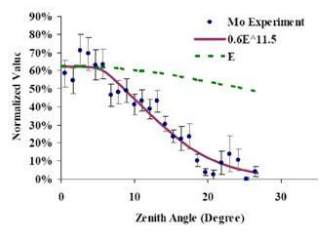

Figure 11: Mo breakdown area fraction vs. zenith angle.

Measurement of the density of pits in the cavity electrode vs polar angle on the hemispherical electrode for three different field and the solid line raises this field to a power nwhich is chosen to fit the data.

Fig.2.14 Data from [13]. The electrode in the cavity was spherical and hence the field varied. The density of pits in the electrode were measured as a function of local field and the function fitted with a power law.

The value $\mathrm{n}$ observed is similar to that observed in vacuum cavities and the peak fields present in the two cases are similar. Field emission has been well verified in vacuum cavities and so we will assume that the surface physics in the two cases at the initiation of breakdown is the same.

Below, we show in Fig.2.13 the index for field emission from tungsten electrodes where we assume the log derivative has been measured to be 10. The red line gives the value the field at the emitter would for the rf case and the green curve uses the correct equations for a DC field. The resulting current density can then be derived from Eq.2.11 or from Fig.2.13. Unfortunately, we only have indirect measurements of $\mathrm{n}$ for the gas filled case and we need to verify that the approach used in [13] represents a valid measure of $\mathrm{n}$, or find a way to measure the field emission directly when high pressure gas is present.

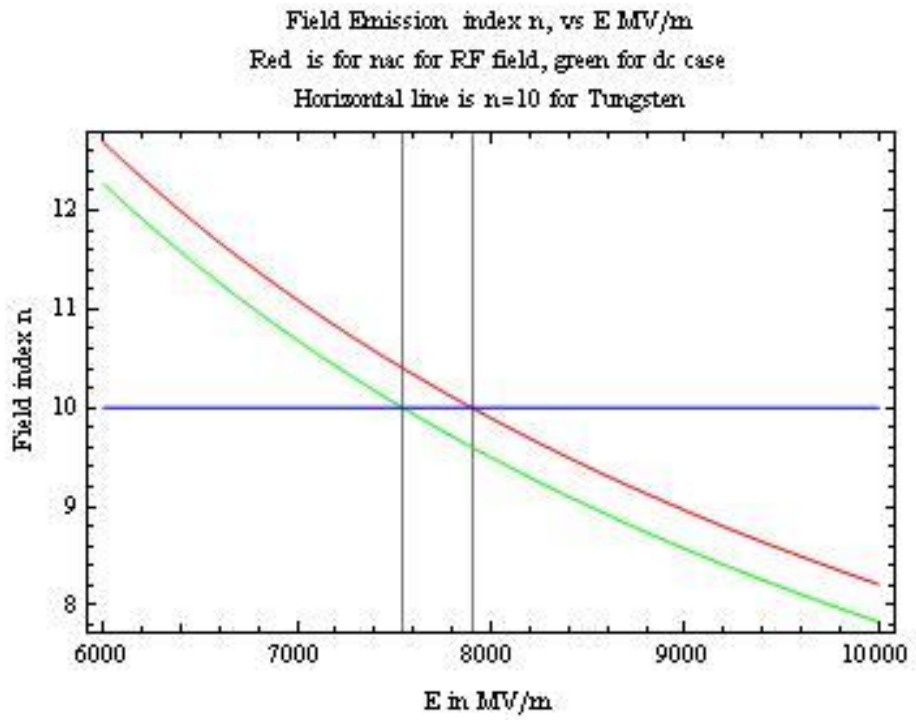

Fig.2.15. The field index as a function of $\mathrm{E}$ for three different cases. The red is the correct expression for RF fields, and the green is for the DC case. The intersection of the measured $n=10$ curves gives the gradient at the emitter and then using Fig2.11, the current density can be found. 
Finally, Fig.2.16 shows the variation of field index with work function. We choose (W, $4.56 \mathrm{eV})$, $(\mathrm{Mo}, 4.60 \mathrm{eV}),(\mathrm{Be}, 4.98 \mathrm{eV})$, and $(\mathrm{Cu}, 4.65 \mathrm{eV})$ as representative elements with different work functions. It is useful to note that the exponent contains $\phi^{1 . .5} / \mathrm{E}$ so that a small percentage in the work function causes roughly1.5 times that caused by the same change in $\mathrm{E}$.

Field index versus RF field

Red Tungsten, wf $=4.56 \mathrm{eV}$

Green Mo, wf $=4.60$

blue Copper, wf $=4.65$

Black Beryllimm, wf $=4.98$

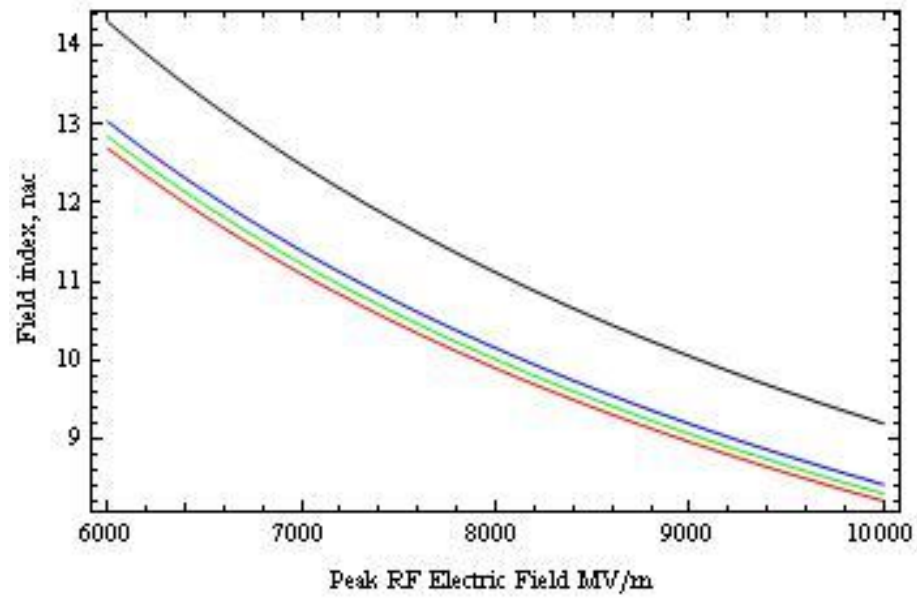

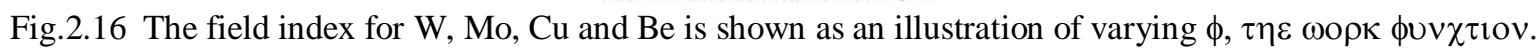

Finally, one can ask are emitters actually seen? The answer is yes [14], and the following two figures are taken from some of the papers that have been written.
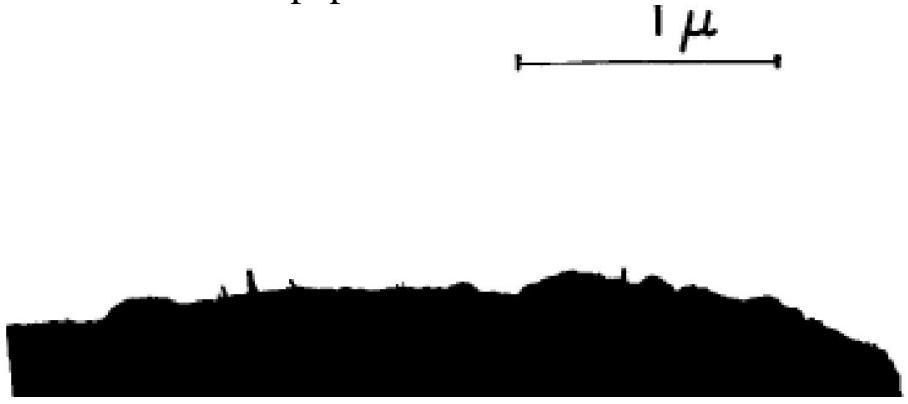

Fig.2.17 Shadow gram of an emitter on a tungsten electrode [14]

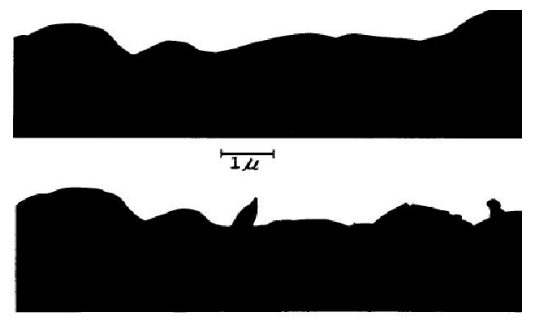

Fig.2.18 Showing the surface of the electrode before and after break down. The emitter was created during the break down process. 
In Fig.2.17, the surface was exposed to a field near break down for about 15 minutes. There is strong evidence that even without actual breakdown that when the field is large that emitters can actually grow on the surface. In the second, Fig.2.18, the shadow gram at the top shows the tungsten surface after being heated to high temperature which left the surface smooth and the bottom image shows the same region after some breakdowns had taken place.

\subsubsection{Emitter geometry and fields.}

Clearly the emitter geometry is complex and poorly defined as can be seen in the figures above.. However there are some cases that we can treat that will give us a feeling for the important parameters. As a first approximation, suppose one has a narrow rod of height $\mathrm{h}$ and radius $\mathrm{r}$ sticking up on a flat surface with a uniform electric field applied. One would guess that it would pull in field lines within a radius comparable to its height. Thus, the average field at the end of the rod would be increased by the ratio of the two areas or by $(\mathrm{h} / \mathrm{r})^{2}$ and an emitter with a ratio of 10:1 can give a field increase of 100. One can actually investigate this a little farther. Using prolate spherical coordinates, we can get the analytical solution for a family of pointed objects. The shapes of these emitters is shown in Fig.2.19

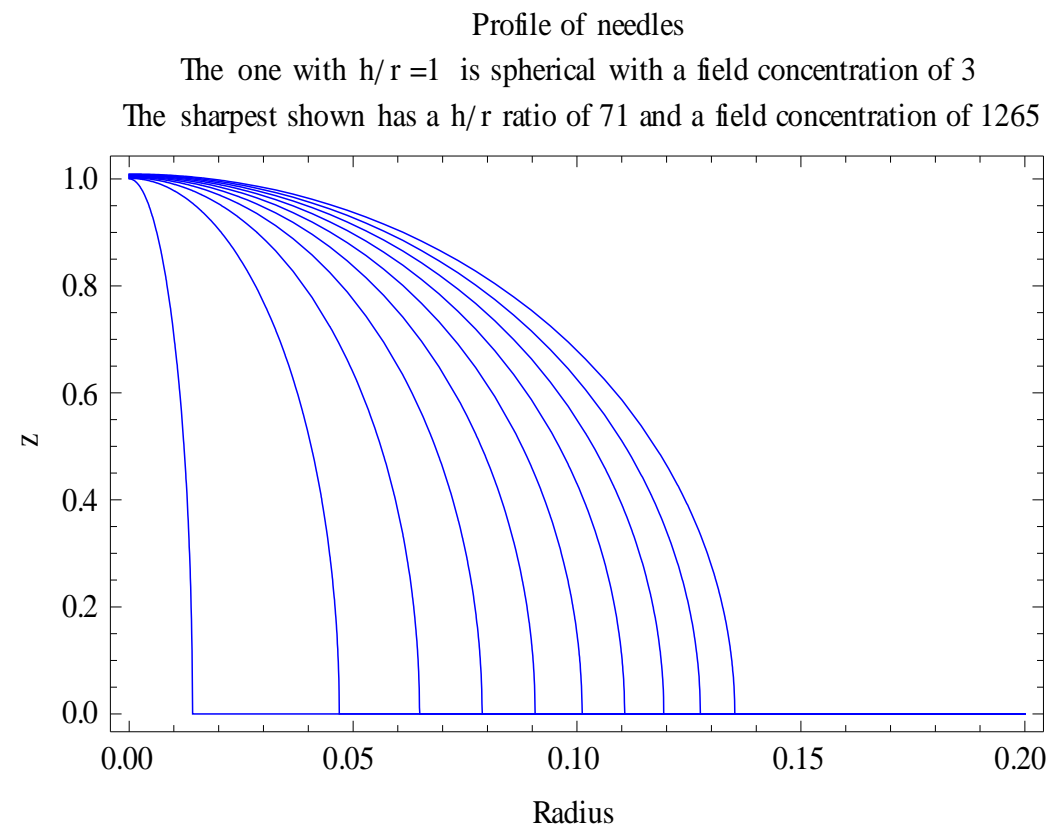

Fig.2.19 This is a family of curves in prolate spheroidal coordinates that have analytical solutions for Laplace's equation. Note the different scales for height and radius. The curve with $\mathrm{h} / \mathrm{r}=1$ is spherical boss and has a field concentration of 3 .

The speculation above that the field enhancement should go as $(\mathrm{h} / \mathrm{r})^{2}$ is verified by the curve in Fig2.20 which shows the field enhancement as a function of this variable. It is seen that a coefficient of 0.5 would make the approximate quite good for $\mathrm{h} / \mathrm{r}>6$. Of course, we have a well defined shape and microscopic changes in the shape will change the local field for distances comparable to the roughness of the point. In any case, $0.5(\mathrm{~h} / \mathrm{r})^{2}$ gives an indication of what the emitter needs to look like for a given field enhancement. 


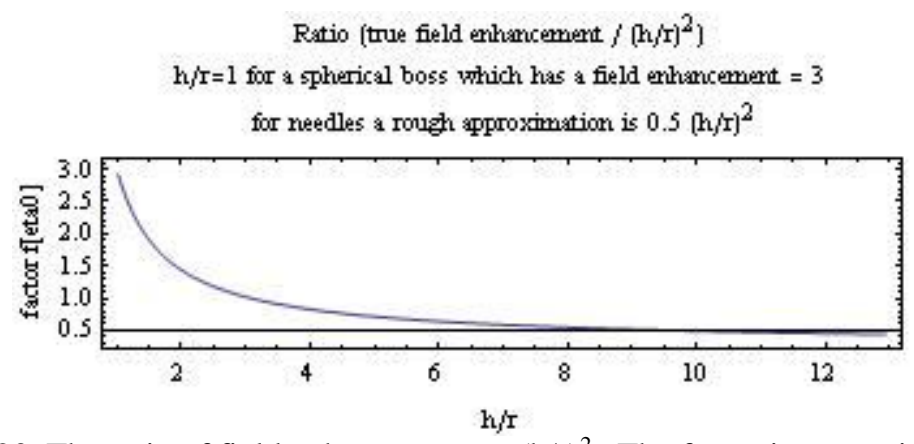

Fig.2.20 The ratio of field enhancement to $(\mathrm{h} / \mathrm{r})^{2}$. The factor is approximately equal to 0.5 for $\mathrm{h} / \mathrm{r}>6$.

An interesting question is how fast does the field fall off and approach the uniform applied field. Fig.2.21 shows an example of an emitter 1.0 micron high with an $\mathrm{h} / \mathrm{r}=18$ that gives an enhancement of 126 . The uniform applied field is $62 \mathrm{MV} / \mathrm{m}$ as shown by the sloping blue line and produces a field at the tip of $7.8 \mathrm{GV} / \mathrm{m}$. The red curve is the potential along the $\mathrm{z}$ axis as one moves away from the emitter tip. The black point is where the field falls below $75 \mathrm{MV} / \mathrm{m}$ and is at 0.4 microns or $40 \%$ of the emitter height. The lower green curve shows 10x expanded horizontal scale for the potential near the tip. We will come back to this figure later.

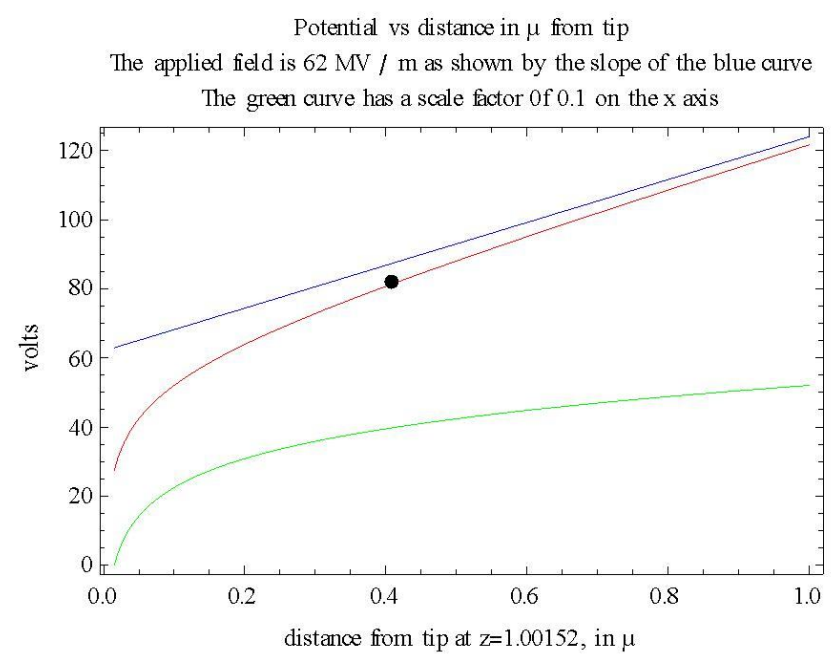

Fig.2.21 The end of the emitter is at $\mathrm{x}=0$ and $\mathrm{V}=0$. The red curve is the electric potential I volts as one moves away along the $\mathrm{z}$ axis and is due to the applied field of $62 \mathrm{MV} / \mathrm{m}$. The blue line gives the slope of the applied field. The green curve is an expanded view of the potential with a factor of 10 on the $\mathrm{z}$ axis. Refering to Fig.1, the black dot is the point where $\mathrm{E}=72 \mathrm{MV} / \mathrm{m}$, which is $\mathrm{E} / \mathrm{P}=14$ for $\mathrm{P}=1000 \mathrm{psi}$ and represents the limiting field for an avalanche to form. The emitter is 1 micron high and has an enhancement factor of 126

\subsubsection{Numerical example for $P=1000$ psia}

We now consider a numerical example: $E=62 \mathrm{MV} / \mathrm{m}$ at 1000 psia with a Paschen limit of 75 $\mathrm{MV} / \mathrm{m}$ and lets consider a 1 micron high emitter for a cavity where $\mathrm{n}=10$ for a tungsten surface. First, $\mathrm{n}=10$ for tungsten yields a field at the tip of the emitter of $7900 \mathrm{MV} / \mathrm{m}$ using the curve in Fig 2.16. Using this value for the field and Fig 2.13 we obtain an emitter current density of $5.410^{6}$ 
$\mathrm{A} / \mathrm{cm}^{2}$. The field enhancement is $7900 / 62=127$ which corresponds to an $\mathrm{h} / \mathrm{r}$ ratio of 15.9 using Fig2.20 which yields a radius of .063 microns. Assuming an area equal to $10 \%$ of the cross section gives a prediction for the total charge injected into the gas per rf cycle of about $0.510^{6}$ electrons. Fig.2.20 has been plotted for just this case and the black dot at a distance of 0.4 microns past the tip corresponds to the point at which the field falls below the Paschen Limit. At this point we need a real simulation to follow what happens. However, we know experimentally from Fig.2.1 that break down does occur. The local field of the electrons and the positive ions that they have produced around the tip raises the local field above the Paschen Limit and the ionization propagates across the cavity. We don't know the details and a complete simulation of the process would be very interesting.

However, this is not a complete description of the breakdown process. If the process described above were the whole story, it would follow that increasing the gas pressure would increase the Paschen limit and the limiting voltage across the cavity would increase. This is clearly not the case...the maximum voltage is independent of pressure! The crucial point is that at a gas density of around $0.005 \mathrm{grms} / \mathrm{cm}^{3}$ there is a transition to a different mechanism of breakdown that is independent of gas pressure but depends on the properties of the metal surface.

\subsection{What causes the plateau?}

At this point we will have to revert to what is being learned from vacuum cavities. They have a big advantage in that the field emission can be measured in this case over many orders of magnitude [6]. If we can find a way to observe field emission in the gas filled case by seeing optical radiation with a phototube, this will be a great help. Or even better would be a cavity that can be operated in both modes would be a great addition.

As mentioned in the previous section, field emission alone cannot explain the plateau. Two processes observed in the vacuum case are at least plausible culprits. The first is that the force from the electric field may actually exceed the mechanical strength of the cavity material and it fractures. The second is thermal stress from heat cycling the material may cause fatigue failure of the material. There are good examples of this type of failure shown in the ANL Workshop [15].

The field necessary to actually tear off atoms from a surface has been studied in field emission microscopes. An interesting paper that gives many details is by Mueller who first exploited the device in about 1960 [16]. The necessary field is of the order of a few volts per Angstrom and is given in the table below taken from [16].

\begin{tabular}{lrl}
\hline Metal & $F^{+}$ & $F^{++}$ \\
\hline $\mathrm{W}$ & 1190 & 530 \\
$\mathrm{Re}$ & 980 & 460 \\
$\mathrm{Ta}$ & $\mathbf{1 1 0 0}$ & $\mathbf{4 5 0}$ \\
$\mathrm{Mo}$ & 795 & 388 \\
$\mathrm{Nb}$ & 800 & 342 \\
$\mathrm{Ir}$ & 955 & 541 \\
$\mathrm{Pt}$ & $\mathbf{7 6 1}$ & 488 \\
$\mathrm{Zr}$ & 700 & 345 \\
$\mathrm{Au}$ & 570 & 505 \\
$\mathrm{Fe}$ & 490 & 352 \\
$\mathrm{Ni}$ & 445 & 385 \\
$\mathrm{Cu}$ & 410 & 475 \\
$\mathrm{Zn}$ & 383 & 388 \\
\hline
\end{tabular}

Table 2.1. The field in $\mathrm{MV} / \mathrm{cm}$ to remove an atom from the surface of various elements is given. Note that the binding depends on the charge state of the ion. This has been measured for a number of elements and it is known that copper is singly ionized and tungsten doubly ionized. 
The fields listed are all a factor of about 5 or more above the field at the tip of an emitter which we know is of the order of $8 \mathrm{GV} / \mathrm{m}$ from the field emission observed in vacuum cavities.

However, metals are notorious in that their tensile strength is well below that predicted from atoms in a perfect crystal and so we should be using the yield or the tensile strength of the material. The following table gives the range of yield strength of various metals and the field that produces these stresses.

Table 2.2. The yield strength and the corresponding electric surface field in $\mathrm{GV} / \mathrm{m}$.

$\begin{array}{lll}\text { Material } & \text { Yield MPa } & \text { Equivalent } \mathrm{E}, \mathrm{GW} / \mathrm{m} \\ \text { Be } & 350415 & \{8.9,9.7\} \\ \text { Al } & 15020 & \{1.8,2.1\} \\ \text { Cu } & 33 & 2.7 \\ \text { SS } & 520 & 10.8 \\ \text { Mo } & 5501150 & \{1.1,16.1\} \\ \text { WT } & 550620 & \{1.1,11.8\}\end{array}$

It should be noted that there is an even wider variation in these numbers than indicated in the table depending on the working of the material during fabrication process. It is interesting that Mo does come out highest, agreeing with measurements.

\subsubsection{Joule heating}

Heating of the asperities or imperfections in the surface may play a role. There are several sources of heating:

1. Joule heating of the emitter from the field emission current

2. RF surface currents

3. Positive ion bombardment of the emitter (not considered here. We need a good simulation in order to address this question.)

4. The final arc that discharges the cavity (see later section)

It is easy to generate a criterion for a lower limit of the necessary current density. Consider a small section of the conductor $\mathrm{dV}$ with density Rho and Heat Capacity $\mathrm{C}(\mathrm{T})$ carrying a current density $\mathrm{j}(\mathrm{t})$. The joule heating minus the cooling can be equated to the increase in temperature

$$
d W=j(t)^{2} / \sigma(T) d t d V-\text { Cooling }=C(T) \text { Rho } d V d T
$$

The cooling can be by conduction or radiation, but to generate a limit, we will set it to zero. We can then write:

Eq. 2.12

$$
\int_{0}^{t} j(t)^{2} d t=\int_{273}^{T} \frac{C(T) R h o}{\sigma(T)} d T
$$


Since the heat capacity and conductivity are known functions of $\mathrm{T}$, the right side of the equation is a function of $\mathrm{T}$ and can be used to give a lower limit on the right side to reach a given temperature. The Fig.2.22 shows this relationship for Tungsten, Moly, Beryllium and Copper and Table[] gives the minimum value for the integral of $\mathrm{j}^{2} \mathrm{dt}$ necessary to reach the melting temperature and also lists the heat of fusion.. Note that this does NOT include the heat of fusion and it neglects cooling. It represents a true lower limit on the integral of $\mathrm{j}^{2} \mathrm{dt}$ to reach the melting point. Cooling by conduction will only increase the requirement. A second point worth noting is that since we have used current density, the answer is independent of the geometry and only refers to the material at any given point.

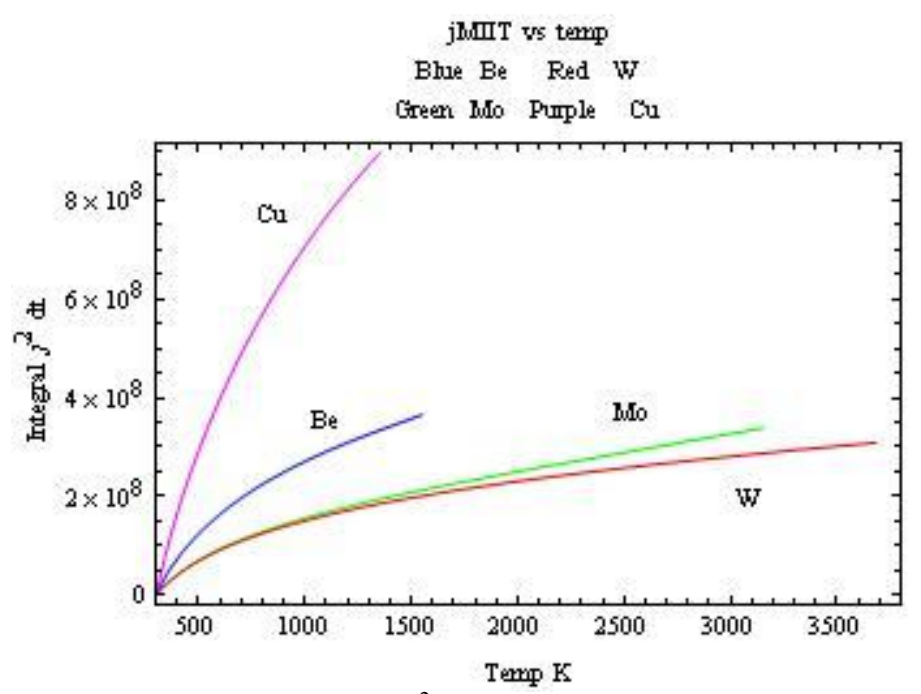

Fig.2.22 This plot shows the integral of $\mathrm{j}^{2} \mathrm{dt}$ necessary to reach the melting point of $\mathrm{W}, \mathrm{Mo}, \mathrm{Be}$, and $\mathrm{Cu}$ under the assumption of no cooling. It also does not include the transition thru fusion. Note that copper is most resistant to melting due to its low conductivity.

Table 2.3. The last column gives Int $\left[j^{2} d t\right]$ to reach melting point. The heat of fusion may have to be added .

$\begin{array}{lllllll} & \text { Density } & \text { M.P. } & \text { Joules to M.P. Heat of Fusion Sum } & \text { Int }\left[j^{2} \mathrm{dt}\right] \\ \text { W } & 19.3 & 3683 & 11602.8 & 3705.6 & 15308.4 & 3.08907 \times 10^{8} \\ \text { Mo } & 10.2 & 3156 & 11064 . & 2927.4 & 13991.4 & 3.38976 \times 10^{8} \\ \text { Cu } & 8.96 & 1356 & 4295.26 & 1818.88 & 6114.14 & 8.96933 \times 10^{8} \\ \text { Be } & 1.84 & 1551 & 6944.55 & 2493.2 & 9437.75 & 3.65227 \times 10^{8}\end{array}$

\subsubsection{Does field emission melt the emitter?}

To answer the above question, we need to integrate $\mathrm{j}(\mathrm{t})^{2}$ over one rf cycle. Note that this is different than the average current over an rf cycle that is shown in Fig[2.13]. The result for tungsten are shown below in Table 2.4 
Table2.4

$\begin{array}{ll}\text { Gradient, NV/m } & \int j a c^{2} \text { dt } \\ 2000 & 1.17642 \times 10^{-18} \\ 2500 & 1.33457 \times 10^{-12} \\ 3000 & 1.59554 \times 10^{-8} \\ 3500 & 0.0000139495 \\ 4000 & 0.00235773 \\ 4500 & 0.132472 \\ 5000 & 3.42923 \\ 5500 & 50.3779 \\ 6000 & 482.857 \\ 6500 & 3326.64 \\ 7000 & 17658.8 \\ 7500 & 76011 . \\ 8000 & 275709 . \\ 8500 & 867963 . \\ 9000 & 2.42675 \times 10^{6}\end{array}$

We note that the values are 3 orders of magnitude or more below that required for melting an asperity consisting of any of the four elements considered above.. The conclusion is that fusion of the emitter or even very strong heating is not important during the very initial phase of breakdown. During the arc phase, there is no doubt that the electrode material gets melted. We return to this subject later.

\subsubsection{Do surface currents cause local heating and breakdown?}

It has also been suggested that joule heating from skin effect surface currents fields may play an important part in starting a breakdown. However, two things are against this proposal. First, the current density is too low to cause melting and second, one notes that the surface current goes to zero at the center of the cavity where the field is highest and most of the break downs are taking place. However, it is known that at very high frequencies and fields (CLIC studies) that the local surface heating of the order of $100^{\circ}$ or less can lead to fatigue, an effect in the metal that disrupt the surface over time and which then does lead to breakdown. Whether or not that is present in our cavities needs to be investigated.

\subsection{4 "Run away electrons"}

At the very high fields achieved in the gas filled cavity, is it possible that the burst of electrons from an emitter in the plateau region could actually be accelerated across the cavity. We develop here the criterion for this to happen:

Eq. 2.13

$$
\frac{d E}{d x} \leq E_{r f}
$$

Where the left side is the loss of energy in the gas and the right is the energy gain from the cavity field. A curve of $\mathrm{dE} / \mathrm{dx}$ for an electron in hydrogen is shown below and has been constructed from a simulation in G4BEAMLINE and some medical data [17].] 


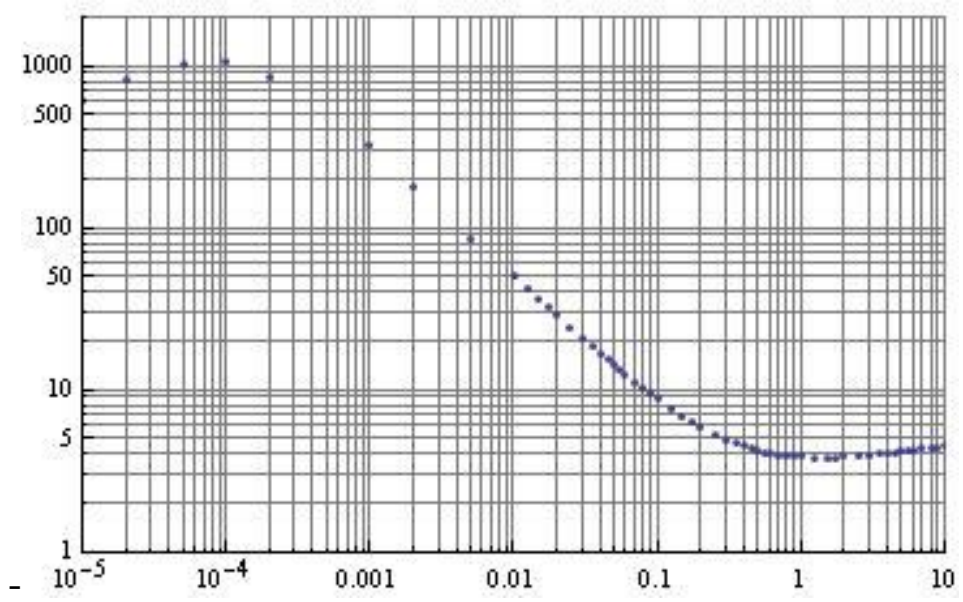

Figure2.23. $\mathrm{dE} / \mathrm{dx}$ for electrons in $\mathrm{H}_{2}$ with density normalized to $1 \mathrm{grm} / \mathrm{cm}^{3}$. The Horizontal scale is in MV and the vertical scale is in $\mathrm{MV} / \mathrm{cm}$.

Consider the Paschen region and pick fields just below the breakdown limit, $\mathrm{E}_{\mathrm{rf}}=14 \mathrm{P}$ we can change $\mathrm{P}$ to density and we can write:

Eq. 2.14

$\mathrm{E}_{\mathrm{rf}}=119 \mathrm{MV} / \mathrm{cm} \times$ density.

Using a density of 1.0, we can pick a point on Fig.2.23 where the accelerating field will be greater than $\mathrm{dE} / \mathrm{dx}$. This point is when the electron energy is greater than $3.3 \mathrm{KV}$. Above this energy the $\mathrm{dE} / \mathrm{dx}$ loss is less than the energy gained from the field and the electron is accelerated across the cavity. This trigger level of $3.3 \mathrm{KV}$ is independent of density along the Paschen limit since $\mathrm{dE} / \mathrm{dx}$ and the maximum $\mathrm{E}_{\mathrm{rf}}$ are both proportional to density. However when the plateau region is reached at a density of .005, $\mathrm{E}_{\mathrm{rf}}$ is fixed and can no longer keep up with the increase in $\mathrm{dE} / \mathrm{dx}$ loss and the minimum $\mathrm{E}$ of the electron to be accelerated must increase. It is clear that the best place to look for these electrons (via the $\gamma$ rays they produce) is at the knee of the curve. At this point the gradient is $0.62 \mathrm{MV} / \mathrm{cm}$ and so there is about $1.8 \mathrm{MV}$ across the cavity. Any run away electrons will have to be at the peak of the rf and a simple integration of the energy gain minus the dEdx loss indicates the electrons will have an energy of the order of $1.5 \mathrm{MeV}$, maximum and their transit time is less than $100 \mathrm{ps}$. It would be interesting to look for these, but the chance of finding any $3 \mathrm{KeV}$ electrons to start the process is very small unless there is some magic going on that we don't understand. With beam, there will be delta rays that satisfy the capture criterion, but their effect will be completely masked by the normal ionization processes.

\subsection{Description of beam transit thru cavity}

We consider first a closed cell cavity with parallel plates and a uniform field and the beam goes thru at the peak of the RF cycle as a delta function. We will pick $\mathrm{dE} / \mathrm{dx}$ for hydrogen as $4 \mathrm{MeV} / \mathrm{grm} / \mathrm{cm}^{3}$ 
and use the measured value of $35 \mathrm{eV} /$ ion pair to calculate the ionization density. A cartoon is shown on the next page, Fig.3.1. The green represents the region of ionization caused by the transit of the beam pulse and immediately after the electrons drift in the field toward the upper plate. The positive ions are so massive that their mobility is very small and they essentially stay in place. As the column of electrons drift upward for $1 / 4$ cycle, they leave a disc of positive ions near the lower plate with thickness deltaz. They also collide with the gas ions and loose energy, making a random walk in the vertical direction. The energy lost in the collision process takes energy out of the field in the cavity. When the rf voltage crosses zero, we will have the situation shown in the next picture, Fig.3.2. Energy has been dissipated and there is a layer of positive charge near the bottom plate equal to the charge of electrons collected on the upper plate of the cavity. During the next half cycle the electrons drift downward leaving an area of positive charge near the top plate and again loosing energy. We should also note that there is an axial magnet field of the order of $5 \mathrm{~T}$ that will confine the plasma in the radial direction. We now need to get quantitative and calculate the magnitude of the ion density and mobility.

Beam passing thru cavity at voltage peak and below that $1 / 4$ cycle later
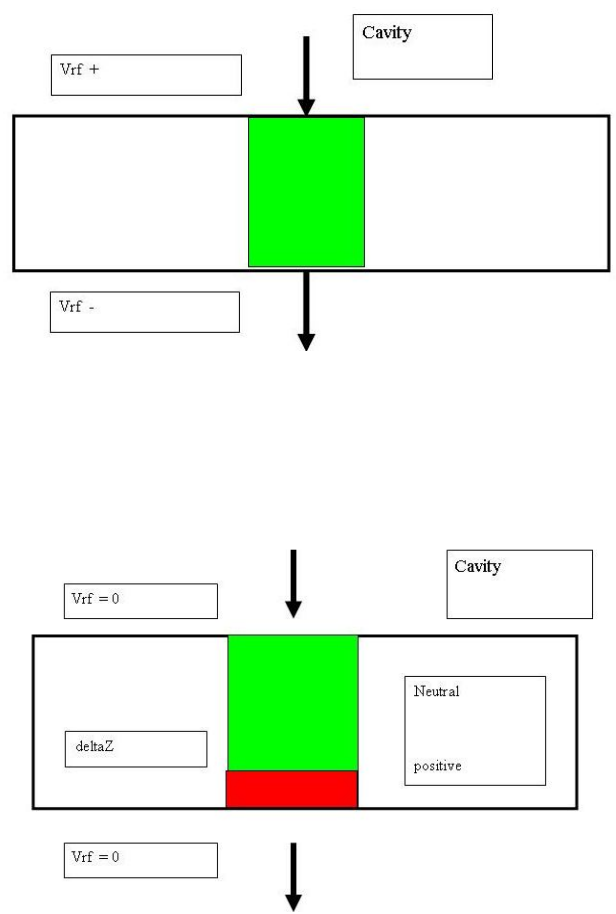

Fig.3.1 The top figure shows the cavity just after a delta function has passed thru at peak field. The green represents ionized hydrogen left behind. The bottom figure shows $1 / 4$ cycle later. The electrons have drifted up leaving behind a layer of positive ions.

The drifting of the electrons represents a current thru the cavity and the inelastic collisions transfer energy from the RF field to the gas molecules. Table 3.1 uses the parameters given below the table to describe a typical state of the cavity after beam transit. The cavity is assumed to be pill box .

Table 3.1 


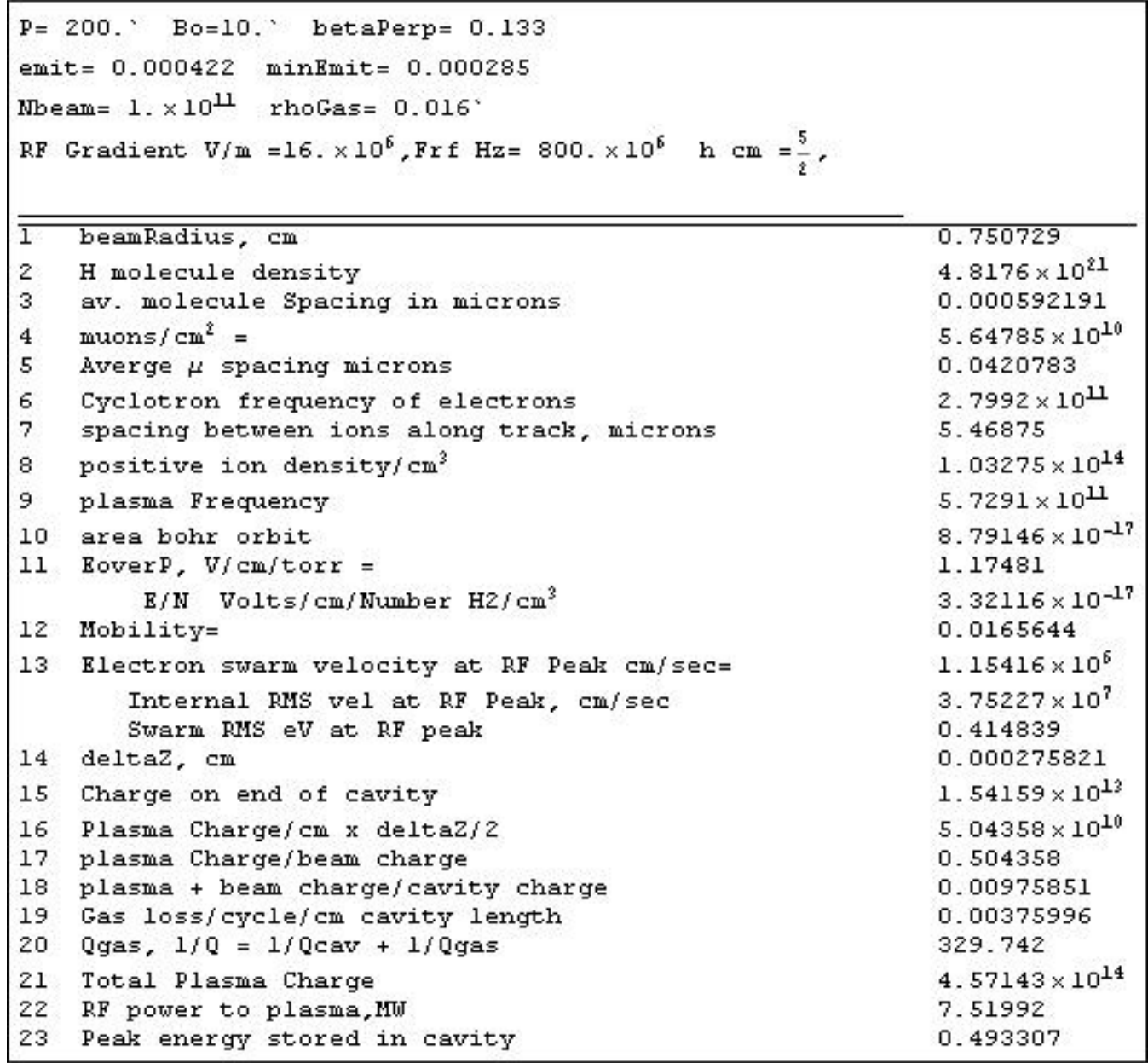

Table 3.1 The above table is an example of the physical environment in a gas filled cavity and can give some feeling for the physical processes taking place. It is important to note that it is assumed that there is no recombination of the electrons and that they remain free to absorb power.

\author{
NOTATION FOR TABLE ABOVE: \\ 1. RMS beam radius \\ 2. Molecular density, grms $/ \mathrm{cm} 3$ \\ 3. Average molecular spacing in microns \\ 4. Density of beam $=$ Beam $/ \mathrm{p}$ muons $/ \mathrm{cm} 2$ \\ 5. Average transverse spacing of muons, microns \\ 6. Radius of 2 eV electron in $\mathrm{B}$ \\ 7. Spacing along track of ionization events \\ 8. Pos ion density/ $\mathrm{cm} 3$ \\ 9. Plasma Frequency, $\mathrm{Hz}$ \\ 10. E / P Volts / cm / P im mmHg \\ 12. Mobility
}

\author{
13. Electron swarm velocity at RF peak \\ Internal RMS electron velocity at RF peak, $\mathrm{cm} / \mathrm{sec}$ \\ RMS electron kinetic energy ar RF peak, eV. \\ 14. Swarm drift distance in $1 / 4 \mathrm{cycle}, \mathrm{cm}$. \\ 15. Electric charge on cavity end, numbr of electrons \\ 16. Plasma charge/ $\mathrm{cm} x$ deltaZ. Number of electrons \\ 17. Plasma charge / Beam Charge \\ 18. (Plasma + Beam)/ Cavity Charge \\ 19. Gas loss/cycle / cm cavity length, Joules \\ 20. Qgas. Use Equation $1 / \mathrm{Q}=1 /$ Qgas $+1 /$ Qcavity \\ 21. Total plasma charge, number electrons \\ 22. RF power to plasma, MW \\ 23. Peak energy stored in cavity, joules
}

The loss to the plasma is calculated as follows:

Eq. $3.1 \quad$ delta $W=\oint i[t] E[t] d x d y d t=\oint\left(v\left[E_{r f} \operatorname{Sin}[\omega t] / P\right] N_{\text {beam }} \rho_{\text {gas }} \frac{d E}{d x} e /\right.$ wip $)\left(.01 E_{r f} \operatorname{Sin}[\omega t]\right) d t$ 
The expression under the integral uses the current derived from the velocity of the electrons and uses the mobility as calculated from Eq.2.10. This loss is used in line 20 to give the Qgas which can be combined with the cavity $\mathrm{Q}_{\text {cav }}$ using $1 / \mathrm{Q}=1 / \mathrm{Qcav}+1 / \mathrm{Qgas}$ to give the total effective $\mathrm{Q}$ after beam passage. This assumes that the electrons remain free and do not get neutralized or form a heavy negative ion. The following curve, Fig.3.1 shows how the gas losses vary vs gas density for four different frequencies for a beam bunch intensity of $10^{11}$.

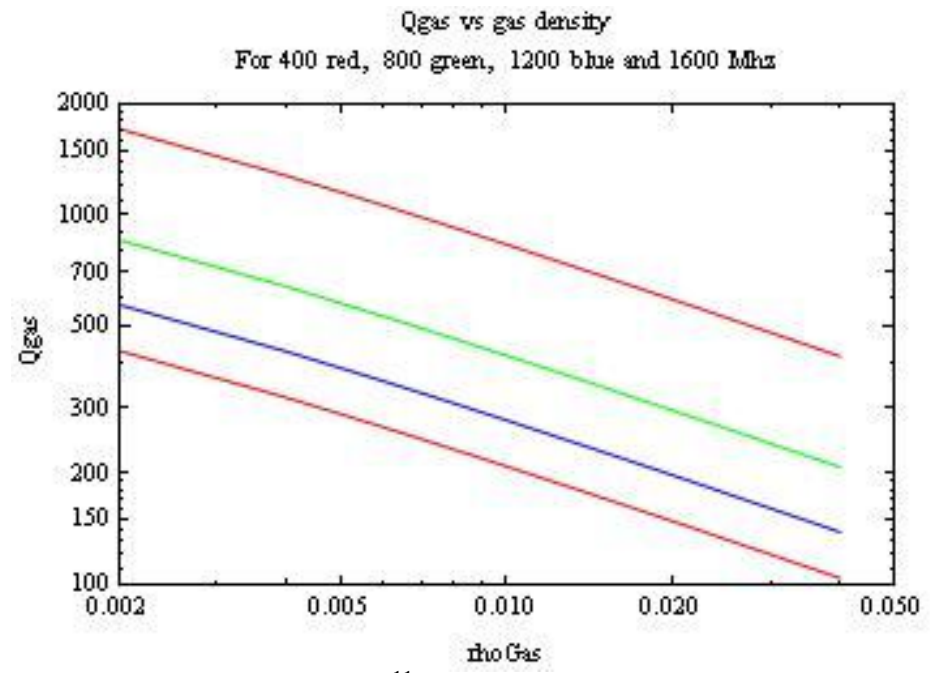

Fig.3.2 This plots the Qgas vs gas density for $10^{11}$ particles. The length of the $400 \mathrm{Mhz}$ cavity is $5 \mathrm{~cm}$ and the length is scaled inversely as the frequency. See the following Table.

The above formulation has been used to calculate the losses in a series of cavities proposed for a Hcc. This channel [27] as proposed has $10^{11}$ beam particles divided into 16 bunches and the frequency is changed as one progressed thru the channel. The table below for the three different frequency cavities is calculated assuming one bunch of $610^{9}$ and also for a total of $10^{11}$ particles which is the situation at the end of the train. The cavity gradient is $16 \mathrm{MV} / \mathrm{m}$.

$\begin{array}{llll}\mathrm{f} & 400 & 800 & 1600 \\ 0 & 12886.3 & 9112 . & 6443.16 \\ \text { Power } & 770016 . & 272242 . & 96252 . \\ \text { mJ/cYcle } & 1.92504 & 0.340302 & 0.0601575 \\ \text { Stored Energy } & 3.94811 & 0.493513 & 0.0616892 \\ \text { Qgas 1 bunch } & 10991.4 & 5495.7 & 2747.85 \\ \text { Qgas 16 bunches } & 659.484 & 329.742 & 164.871 \\ \text { TotalPower@10 } & 1.577 \times 10^{7} & 7.79224 \times 10^{6} & 3.85625 \times 10^{6}\end{array}$

Table.3.2 Effect on beam transversal of 3 different pillbox cavities in an hcc channel. The cavity lengths are 5, 2.5, $1.25 \mathrm{~cm}$. Note the Qgas line. Even at $10^{11}$ muons, the beam loading can be a serious problem unless there is some way to either neutralize the electrons or attach them onto a heavy ion such as $\mathrm{SF}_{6}$.

In addition to the power dissipated in the gas, the charge collected from the plasma increases the effective charge of the beam. This is shown in line 16 in the above table. The immediate effect is to reduce the voltage across the cavity by $\delta q / C_{\text {effective }}$ where the capacity is calculated from:

Eq. 3.2

$$
C_{\text {eff }}=2 \text { EnergyStored } /\left(h E_{r f}\right)^{2}
$$


This beam loading, $\delta \mathrm{V} / \mathrm{V}$, is shown in the figure below for the four different cavities in the plots above.

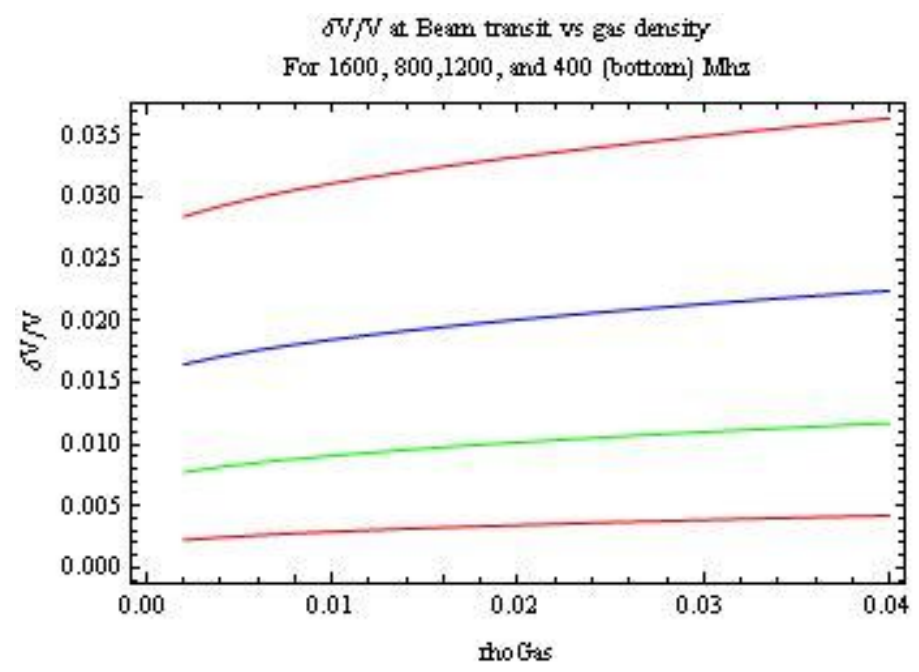

Fig.3.3. Change in cavity voltage with beam passage vs. gas density for four different frequencies, $10^{11}$ particles, pillbox cavities operating with a gradient of $16 \mathrm{MV} / \mathrm{m}$. The $400 \mathrm{Mhz}$ cavity is $5 \mathrm{~cm}$ long and the lengths are scaled inversely as the frequency.

It is clear from the above figures that loading of the cavity from the plasma can be a serious problem as the beam intensity increases. This could become prohibitive in the initial capture channel where there are many protons and pions in addition to the muons. See [26].

\subsection{Recombination of electrons}

The question of recombination of the plasma has to be addressed. If the recombination is extremely fast, the effects discussed above will be absent and if it is very slow, it can influence subsequent cycles. In addition to straight recombination, we will also consider adding a gas such as $\mathrm{SF}_{6}$ which can capture the electrons. If one can quickly attach an electron onto either $\mathrm{H}$ or some other heavy molecule, then the cavity loading calculated above will not happen. We still have to face the question of what happens ultimately to the mixture of heavy charged ions, but initially, we have solved the cavity loading by the nailing down the electrons.

For a two body process, the recombination rate is defined by the equation

Eq. 4.1

$$
\mathrm{dn}_{1} / \mathrm{dt}=\mathrm{R} \mathrm{n}_{2} \mathrm{n}_{1}
$$

where $\mathrm{n}_{1}$ and $\mathrm{n}_{2}$ are the concentrations of the two species participating in the reaction. Note that if $\mathrm{n}_{2}=\mathrm{n}_{1}$, as is the case for hydrogen ion recombination, the equation can be integrated:

Eq. 4.2

$$
\mathrm{n}_{1}(\mathrm{t})=\mathrm{n}_{1}(0) /\left(1+\mathrm{n}_{1}(0) \mathrm{R} \mathrm{t}\right)
$$

This is not exponential but a time scale for the initial disappearance rate of the process is $\mathrm{Rn}_{1}$. It has a $1 / t$ tail and ions can last for very long times. On the other hand if $n_{2}$ represents some species that eats electrons and if $n_{2} \gg n_{1}$ then the decay is exponential with a rate given by $r n_{2}$. If electrons are 
to disappear in the order of a nanosecond, then for a density of $10^{14}-10^{15}$, which is in the range of interest, the recombination rate must be of the order of $10^{-5}$ or even greater. This implies a large cross section for electron attachment of the order of $10^{-16} \mathrm{~cm}^{2}$ or greater.

It is useful to digress and discuss how $\mathrm{r}$ is determined. First it is specific to the interaction type, such as recombination, ionization, excitation, momentum loss, etc. We define two related quantities that will be useful. Consider particle moving thru the plasma and striking particle type 2 and making collisions of type $\mathrm{x}$ with a cross section $\sigma_{\mathrm{x}}$

Eq. 4.3

$$
\mathrm{dN}_{1}=\sigma_{\mathrm{x}} \mathrm{N}_{2} \mathrm{dz} \quad \text { divide by dt to get }
$$

$$
\mathrm{dN}_{1} / \mathrm{dt}=\mathrm{v}_{\mathrm{x}}=\left\langle\sigma_{\mathrm{x}} \mathrm{V}_{1}>\mathrm{N}_{2}=\mathrm{R}_{\mathrm{x}} \mathrm{N}_{2}\right.
$$

Where $R_{x}$ is the rate and $N_{1}$ is the number of interactions of type " $x$ " the incident particle has in going a distance $\mathrm{dz}$ thru type $\mathrm{N}_{2}$. Note that $\mathrm{V}_{1}$ is the velocity of the incident particle. If we are considering electrons as the incident particle then it is not the swarm drift velocity, but rather the internal velocity within the swarm. See Eq. 2.10a. If it is collisions between molecules or ions, then it is the temperature of the gas that determines the velocity. The second variable, $v_{\mathrm{x}}$, is the "collision frequency" for reaction type " $x$ ". The collision frequency for "momentum loss" is used in the literature to calculate the plasma losses in a RF cavity filled with plasma [18]. The quantity $\left\langle\sigma_{\mathrm{x}} \mathrm{V}_{1}\right\rangle$ requires the velocity distribution function in order to be evaluated but can also be estimated from information on the cross section. Some cross section curves are given below.

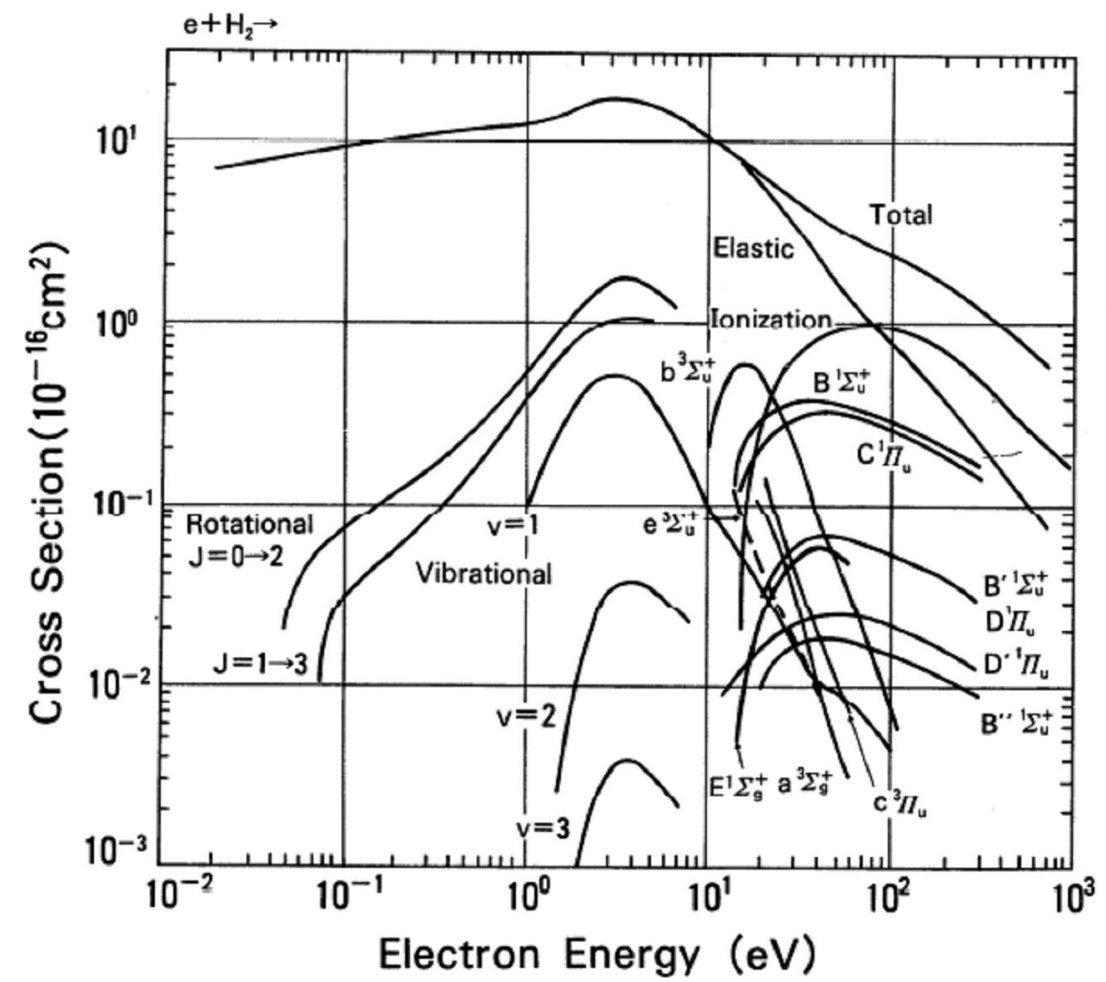

Fig.4.1. Comparisons of various cross sections for e $+\mathrm{H}_{2}$ as well as some atomic values. See Ref.[19]. Note that the peak cross section for ionization is about $10^{-16} \mathrm{~cm}^{2}$ but drops very rapidly to the threshold at $15.3 \mathrm{eV}$. $\{19\}$ 
We will now show an example for the beam-cavity case shown in Table 3.1. The following Fig. 4.2 shows a preliminary result of some rate calculations using the information available in the literature.

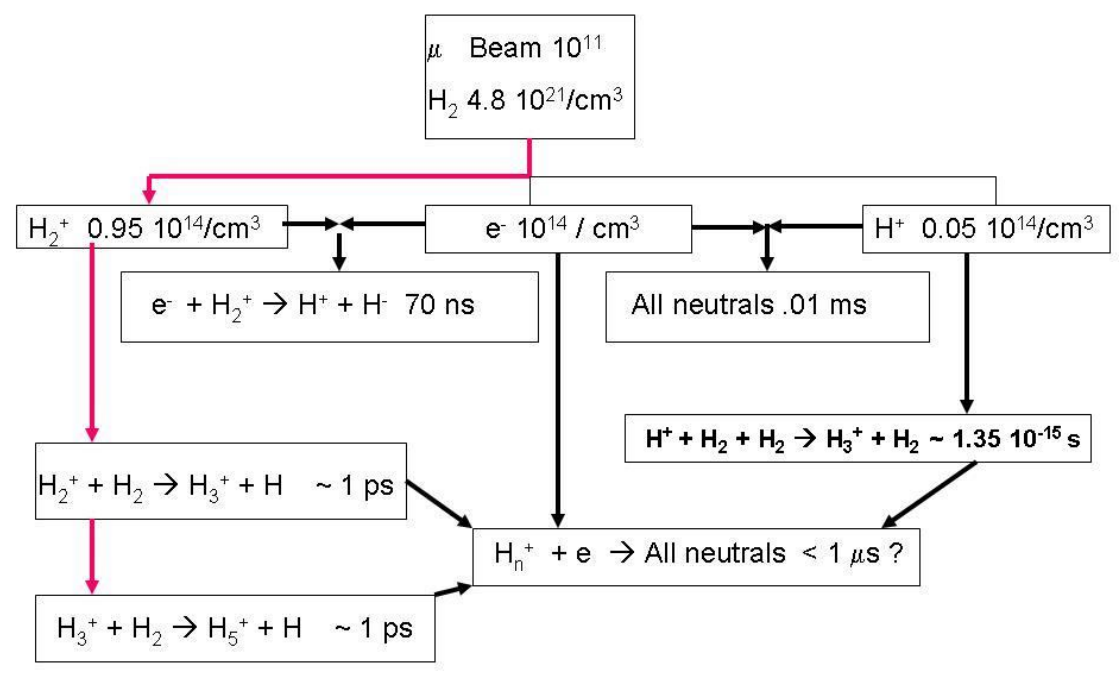

\section{Hydrogen ion chemistry}

Fig. 4.2. The beam-cavity initial condition is shown in the box at the top and the red line shows the predicted path for the evolution of the $\mathrm{H}_{2}{ }^{+}$ion.

The beam produces ions in the $\mathrm{H}_{2}$ gas that are $95 \% \mathrm{H}_{2}^{+}$and about $5 \% \mathrm{H}^{+}$, and an electron density of $10^{14}$ electrons $/ \mathrm{cm}^{3}$ which is equal to the sum of the two species. The state of the electron gas can be calculated using the tools we have developed for calculating mobility plus the cross section data in Fig. 4.1. The first question concerns the relaxation time of the swarm. If it is very small compared to the RF period, the temperature of the swarm will follow from eq.2.10 and will move up and down at twice the RF frequency. We will show that this is the case:

\section{From Table 3.1}

rms internal swarm velocity $=2.610^{7} \mathrm{~cm} / \mathrm{sec} ;$ internal swarm energy $=0.2 \mathrm{eV}$;

$$
\mathrm{e} \text {, density } 1.0310^{14} / \mathrm{cm}^{3} ; \mathrm{H}_{2}, 4.810^{21} / \mathrm{cm}^{3}
$$

From Fig. 4.1 we see that the elastic cross section dominates and is $10^{-15} \mathrm{~cm}^{2}$ at an energy of $0.2 \mathrm{eV}$. Thus the collisions/sec $=2.610^{7} 4.810^{21} 10^{-15}=1.2510^{14}$. The swarm energy is $200 \mathrm{mV}$ and in the average collision, $2 \mathrm{~m}_{\mathrm{e}} / \mathrm{M}$ of this energy is lost. So $\mathrm{dE} / \mathrm{dt}=1.2510^{14} \mathrm{E} / 1800$ or a time constant of about 14 ps. This is a rough calculation but shows the method. If one is more careful and includes the rotational states, their population at room temperature and the energy loss in excitation, the relaxation time is about 3 ps. This is an important result as it means that the mobility and hence the velocity follow from Eq. 3.9 using the instantaneous value of the RF voltage. It also means that any interactions involving the electrons with other molecules must be averaged over the RF cycle properly. This is not a trivial point as some of the molecular cross sections vary over large ranges between thermal energies and 1 or $2 \mathrm{eV}$. 
The main chain of the subsequent interaction between the $\mathrm{H}_{2}{ }^{+}$ions, the $\mathrm{H}_{2}$ molecules of the gas and the electrons is shown by the red line. Most cross sections are of the order of $10^{-16}-10^{-15} \mathrm{~cm}^{2}$ and since the density of the hydrogen molecules is almost $10^{7}$ times that of the electrons, the ions react with the ions and the chain of hydrogen clusters is set up: $\mathrm{H}_{2}{ }^{+}, \mathrm{H}_{3}{ }^{+}, \mathrm{H}_{5}{ }^{+}, \ldots . . \mathrm{H}_{11}{ }^{+}$! The reaction rate for

Eq. 4.4

$$
\mathrm{H}_{2}^{+}+\mathrm{H}_{2} \rightarrow \mathrm{H}_{3}^{+}+\mathrm{H}
$$

is estimated from the measurements in Ref. [20,21]. It has a large cross section for dissociation with electrons, but this cross section may be suppressed at high pressure [22]. If this is the case, then the $\mathrm{H}_{3}{ }^{+}$will be absorbed in the following three body reaction.

Eq. 4.5

$$
\mathrm{H}_{3}^{+}+\mathrm{H}_{2}+\mathrm{H}_{2} \rightarrow \mathrm{H}_{5}^{+}+\mathrm{H}_{2}
$$

These clusters have been observed up to $\mathrm{H}_{11}{ }^{+}$with decreasing $\mathrm{Q}$ value [23]. They represent the positive ion population that is available to eat the electrons. The measured cross section for dissociation into all neutrals have been measured for $n=3$ and $n=5$ at low pressure [24] and give neutralization times of the order of 1 microsecond or less as shown in Fig 4.2. At this point we seem to be in unknown territory as there has been no work done at high pressure since the main drive is from the fusion and astrophysics communities.

\subsection{Use of an electronegative gas.}

The discussion above has centered on neutralizing the electrons by capture on positively charged hydrogen ions. There is a second way to reduce the electron loading and that is to capture the electrons on an electronegative gas such as $\mathrm{SF}_{6}$. The mobility of the ions is so small that the induced RF current is a negligible load. The radiation length of $\mathrm{SF}_{6}$ is $28.87 \mathrm{grms} / \mathrm{cm}^{3}$. If we degrade the radiation length of hydrogen by $1 \%$, the molecular ratio is $6.510^{-5}$. The recombination rate for $\mathrm{SF}_{6}$ has been measured for electrons and gas at $300 \mathrm{~K}$ and is $2.710^{-7}$ [22] and falls with increasing electron temperature. Now for the example we are using (Table 4.1) the electron temperature is much higher than $300 \mathrm{~K}$ at peak voltage, but as the voltage cycles thru zero, the electron temperature drops as we have seen above. If we take the recombination rate equal to $10^{-7}$ the electron capture time is of the order of $0.1 \mathrm{~ns}$ which essentially removes them from play. $\mathrm{SF}_{6}$ has some disadvantages in that its reaction products are very corrosive and it freezes at $\mathrm{LN}_{2}$ temperatures. Better gases need to be found!

\subsection{What happens to the ions?}

We need to answer the question of how long does it take to clear the residual ions after the beam has passed thru the cavity. The total number of ion pairs formed during the passage of $10^{11}$ muons thru $2.5 \mathrm{~cm}$ of $\mathrm{H}_{2}$ with a density $.016 \mathrm{grms} / \mathrm{cm}^{3}$ is 85 micro coulombs. To sweep this total charge out in 
$1 \mathrm{~ms}$ would take a current of $85 \mathrm{ma}$, but this is not reasonable considering the low mobility of the heavy ions. Consider a DC sweep field strength of $1000 \mathrm{~V} / \mathrm{cm}$ and a volume of plasma near the center of the cavity. The field lines must terminate on charges and the charge required is equal to

$\varepsilon_{0} \mathrm{E}=5.510^{8}$ ions $/ \mathrm{cm}^{2}$. This is much smaller than the density present so the field only penetrates a very small distance and slowly peels of the outer layer of ions. Furthermore the mobility is much smaller than the electrons and so their velocity is low limiting the current to small values. If the plasma is $\mathrm{H}^{+}$and e the rate of sweeping is still limited by the velocity of the hydrogen ion as the plasma wants to stay neutral.

This is a problem that at present we don't have a good way of measuring. If nothing else works, one could consider circulating the gas fast enough to remove the ions between pulses. This is a subject for more work!

\subsection{The Discharge}

When the discharge finally occurs, the cavity is discharged in a very short time. There is no question that copper is melted or vaporized and form additional emitters on the surface. With a little analysis, we can learn something about the conditions during this phase, although one would rather that such discharges could be prevented!

For this analysis, we use a simplified circuit of the cavity. The coupling to the drive is weak enough so that during the actual discharge we can neglect its effect. For longer times it does have an effect and may even re-strike the arc. Consider the following diagram of the pillbox shaped test cavity:

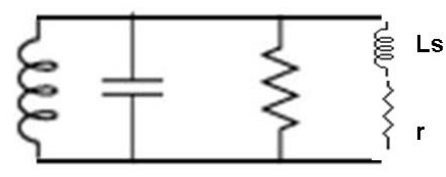

L $\quad$ C $\quad R$

Figure 5.1 Assumed equivalent circuit of the cavity with a spark in it.

The value for $\mathrm{L}$ was obtained by the total energy stored in the cavity as calculated by Mohammad Alsharo'a [25] and equating that to $1 / 2 \mathrm{LI}^{2}$. C was then chosen to make the resonant frequency correct. R is given by the measured $\mathrm{Q}$, but once the arc starts there is a parallel circuit with Ls and $\mathrm{r}$ that represent the spark channel. The Ls depends primarily on the radius of the arc and its length and the value of $r$ is dependant on the very hot plasma in the arc.

Eq. 5.1

$$
L s=\frac{\mu_{o}}{2 \pi} h \log [r 1 / r 2]+\frac{\mu_{o}}{8 \pi} h
$$

Where Ls is the inductance of the spark channel, $\mathrm{r} 1$ is the cavity radius, $\mathrm{r} 2$ is the channel radius, and $\mathrm{h}$ is the length of the spark. $\mathrm{L}$ is $24.2 \mathrm{nh}, \mathrm{C}$ is $1.61 \mathrm{pf}$ and we will neglect $\mathrm{R}$ as the cavity $\mathrm{Q}$ is large. The figure below shows the spark channel inductance vs its radius in microns. 


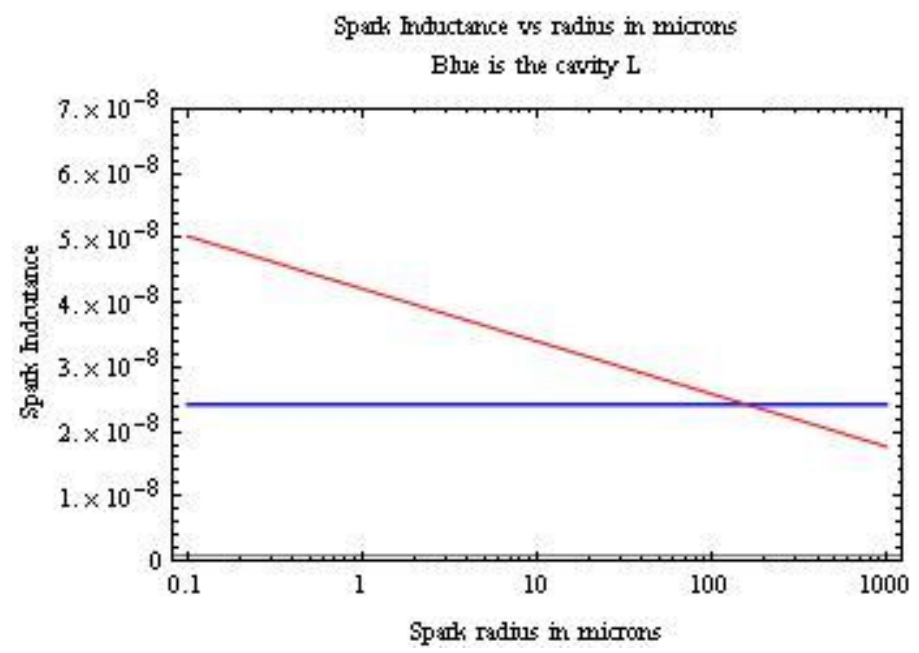

Figure 5.2 Red is the spark channel inductance vs. its radius in microns and the blue is the cavity inductance.

It's clear from the above figure that as the channel inductance can influence the resonant frequency of the discharging cavity and the $\mathrm{Q}$ will give some indication of the resistance. This is a complicated dynamic process as the channel is compressed by the large self magnetic field (pinch) and at the same time has a high pressure due to the heating of the plasma. We solve the above circuit and realize that we will only get some kind of average value for radius. The Laplace Transform for the circuit is given by:

Eq. 5.2

$$
z[p, x, r]=\frac{1}{\frac{1}{p L}+p C+\frac{1}{(x p L+r)}}
$$

Where we have used $\mathrm{x}$ as the ratio of Ls / L. If we apply a delta function, Qo of current to this Z, it charges the $\mathrm{C}$ to a voltage Qo/C and we arrange Qo to give the initial voltage across the cavity when the breakdown starts. The curves on the next page show the voltage across the cavity for various size damping resistors for a nominal value of the spark radius of 

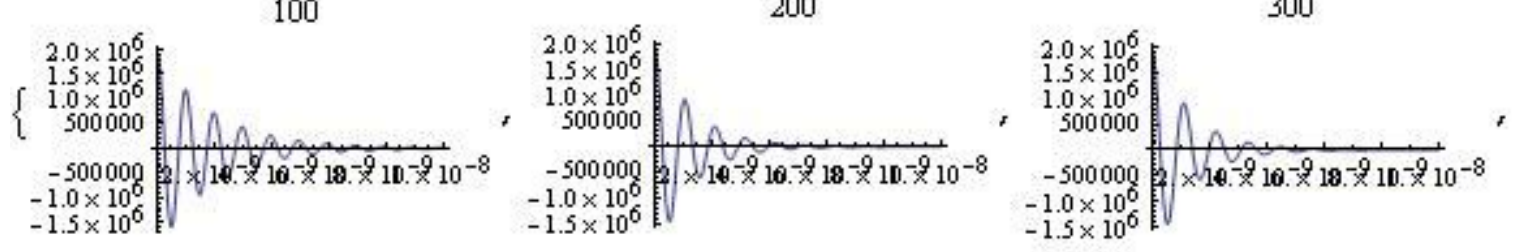

400

500
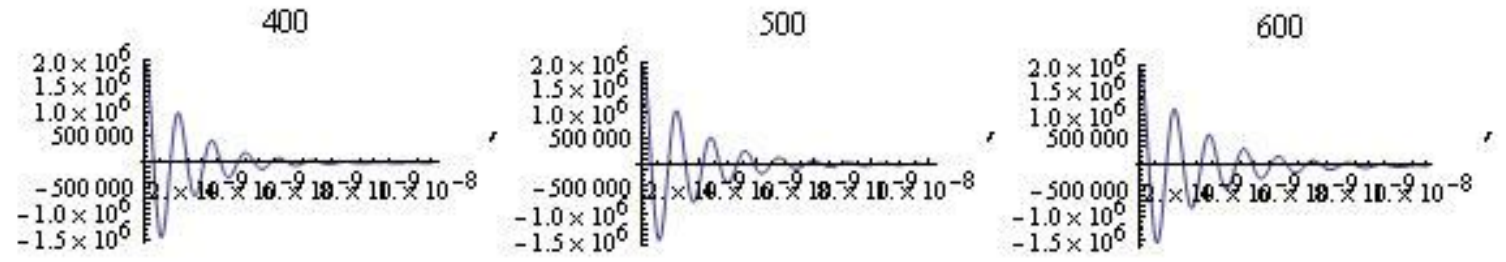

700

800

900

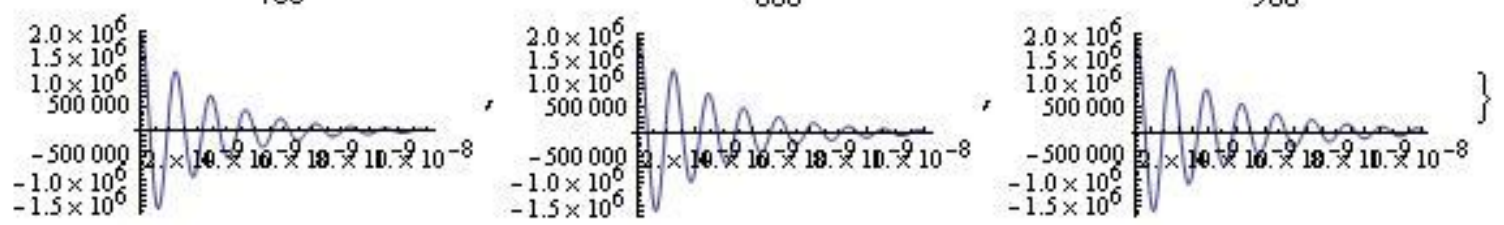

Figure 5.3 Cavity voltage waveforms for different values of the spark channel resistance. The spark inductance is fixed at 1.5 times the cavity inductance. The initial voltage is $210^{6}$ volts.
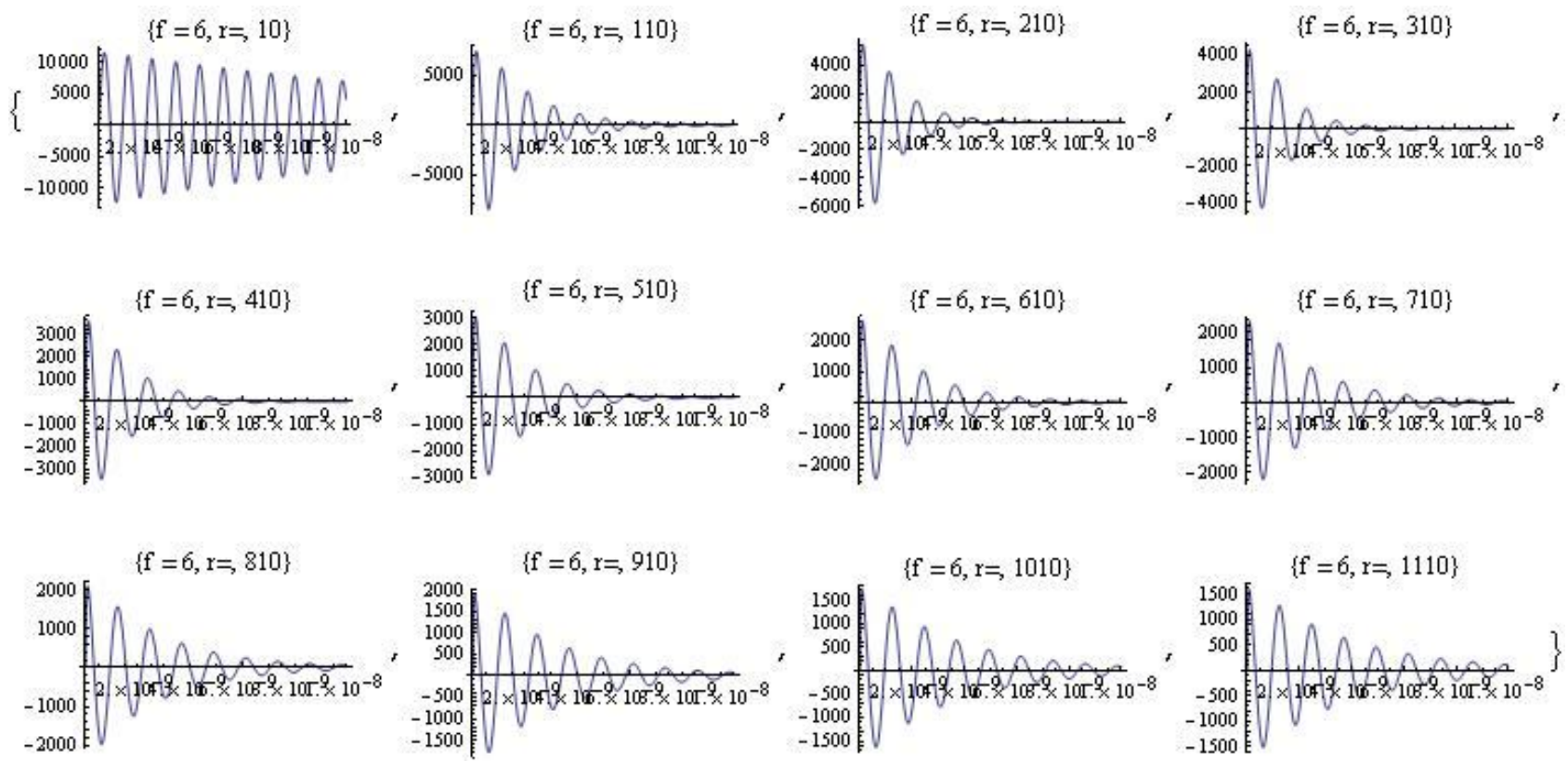

Figure 5.4 Spark channel current for various values of the channel resistance. The inductance is fixed at 1.5 times the cavity inductance. The initial voltage is $210^{6}$ volts. 
As the spark forms, its radius changes and hence its inductance. This will show up as a change in the cavity resonant frequency. We can show the relation between the damping time and the resonant frequency in the following

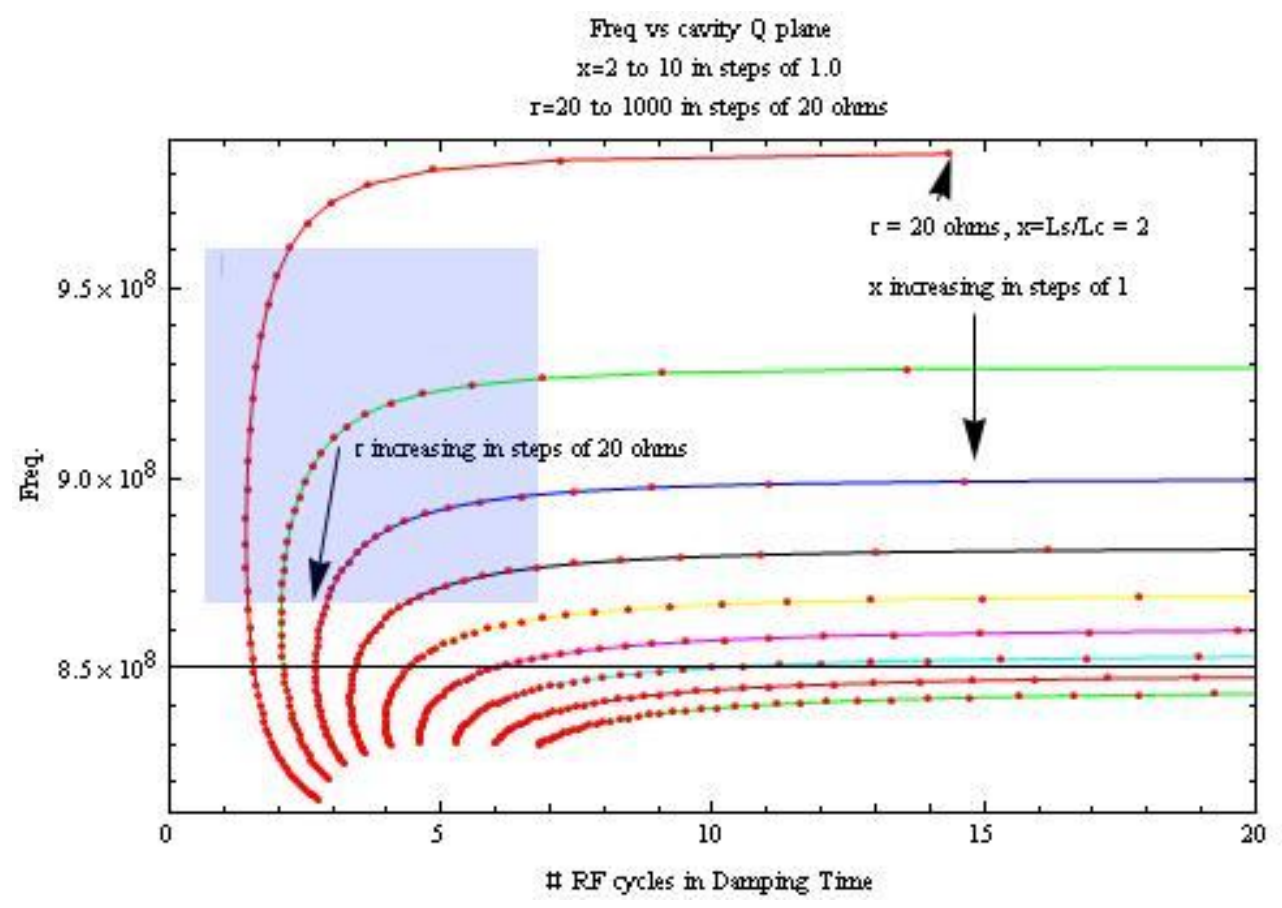

Figure 5.5 Cavity resonant frequency vs. damping time for different values of $\mathrm{r}$ and $\mathrm{x}=\mathrm{Ls} / \mathrm{Lc}$. Events have been observed in the shaded area.

One can understand the above diagram in the following way. When $r$, the spark channel resistance is very low, the channel inductance is placed in parallel with the cavity inductance and the frequency is increased. This is the situation in the upper right portion of the diagram. On the other hand when $r$ is large, the current is limited and the inductance cannot shift the frequency which is the case in the lower portion. Yonehara has made a measurements looking for the frequency shift and measuring the damping time. Events have been observed in the shaded area which imply spark channels that have a radius of the order of $1 \mu$ and resistances of the order of a few 100 ohms.

\subsection{Some things we would like to know}

1. In the plateau region what is the mechanism that initiates the breakdown. Is it a fracture of the asperity? Is it rapid heating due to positive ion bombardment? Or something else?

2. Can we see light being emitted before the spark? Do $\mathrm{H}_{2}{ }^{+}$and $\mathrm{H}^{+}$get eaten by $\mathrm{H}_{2}$ molecules and turned into $\mathrm{H}_{3}{ }^{+}$which decays to all neutrals.

3. How many RF cycles does it take the streamer to cause break down and what is the $\mathrm{H}$ ion chemistry in the streamer path.

4. How long do the positive and negative ions hang around and do they help initiate breakdown at later times with succeeding pulses? Can we sweep them out and does it matter?

5. Can we find electronegative gases that don't freeze out and don't form nasty byproducts? 
6. The collision frequency is much higher than the cyclotron frequency which means in principle that the gas filled cavities are immune to the effect of a magnetic field. Is this really true with beam or is there something we have missed?

7. Are there gases that will keep the electron temperature low and thus the mobility low. This may be a solution that is different than the one using electronegative gases.

8. This is a good start!

\section{References}

1. P. Hanlet et al., "STUDIES OF RF BREAKDOWN OF METALS IN DENSE GASES", PAC06, Knoxville, Tennessee.

2. D. J. DeBritetto and L. H. Fisher, Physical Review 104, 1213 ,(1956).

3. J. T. Tata and P. T. Smith Physical Review 19, 270,(1932)

4. See D. V. Ross. "Low Emittance Workshop, FNAL, April 21-25 2008"

5. J. L. Pack and A. V. Phelps Physical Review 121, 798, (1960).

6. A. Bartels, PRL 28, 213, (1971).

7. A.E.D. Heylen, Proc. Roy Soc. 76, 779 (1960)

8. J. Norem, et al. PRST 6, 072001-1, (2003).

9. A. Moretti et al, PRST 8, 072001-1,(2005)

10. A. Hassanein et al, PRST 9, 062001-1, (2006)

11. E. L. Murphy and R. H. Good, Jr., Physical Review, 102, 1464, (1956). This paper covers also the transition region where the temperature is high enough for thermionic emission.

12. H. E, Burgess and H. Kroemer, Physical Review, 90, 515, (1953)

13. M. BastaniNejad et al, PAC07 Albuquerque, paper WEPMS071

14. H. E. Tomaschke and D. Alpert, Journal Vacuum Science and Technology,4, 192,(1967)

15. Agenda for High-Gradient Collaboration Workshop (ANL, March 3-4, 2009) http://www.hep.anl.gov/ushighgradient/tentative-agenda.pdf

16. E. W. Muller, Science 149, 591, 1965. 
17. Yonehara, K. private communication and H. Sugiyama, Phys. Med. Biol., 1985, Vol 30, 4, 331-335

18. Moses Chung, et al MuCool Note NFMCC 532-v2

19. H. Tawara et al, J. Phys. Chem. Ref. Data, 19,617,(1990)

20. R. H. Neynaber and S. M. Trujillo PR 107, 167, (1967)

21. Clayton F. Giese and William B. Maier II, J. Chem. Physics, 39, 739, (1963)

22. V. Kokoouline and C.H. Greene, PR A, 68, 012703-1, (2003)

23. K. Hiraoka and P. Kebarle, Jour. Chem. Phys. 62, 2267, (1975)

24. J. Glosik, et al., arXiv:0903.1656v1 \{physics.plasm-ph \} 9 March 2009

25. Mohammad Alsharo'a, MuonsInc. Private communication.

26. Moses Chung et al NFMCC-doc-532-v2.

27. Yonahara, $\mathrm{K}$. private communication, but available at the following url http://www.fnal.gov/directorate/Fermilab_AAC/AAC_July_07/Talks/AAC_HCCSimulation_K Y_august8_07_final.ppt.

\section{APPENDIX}




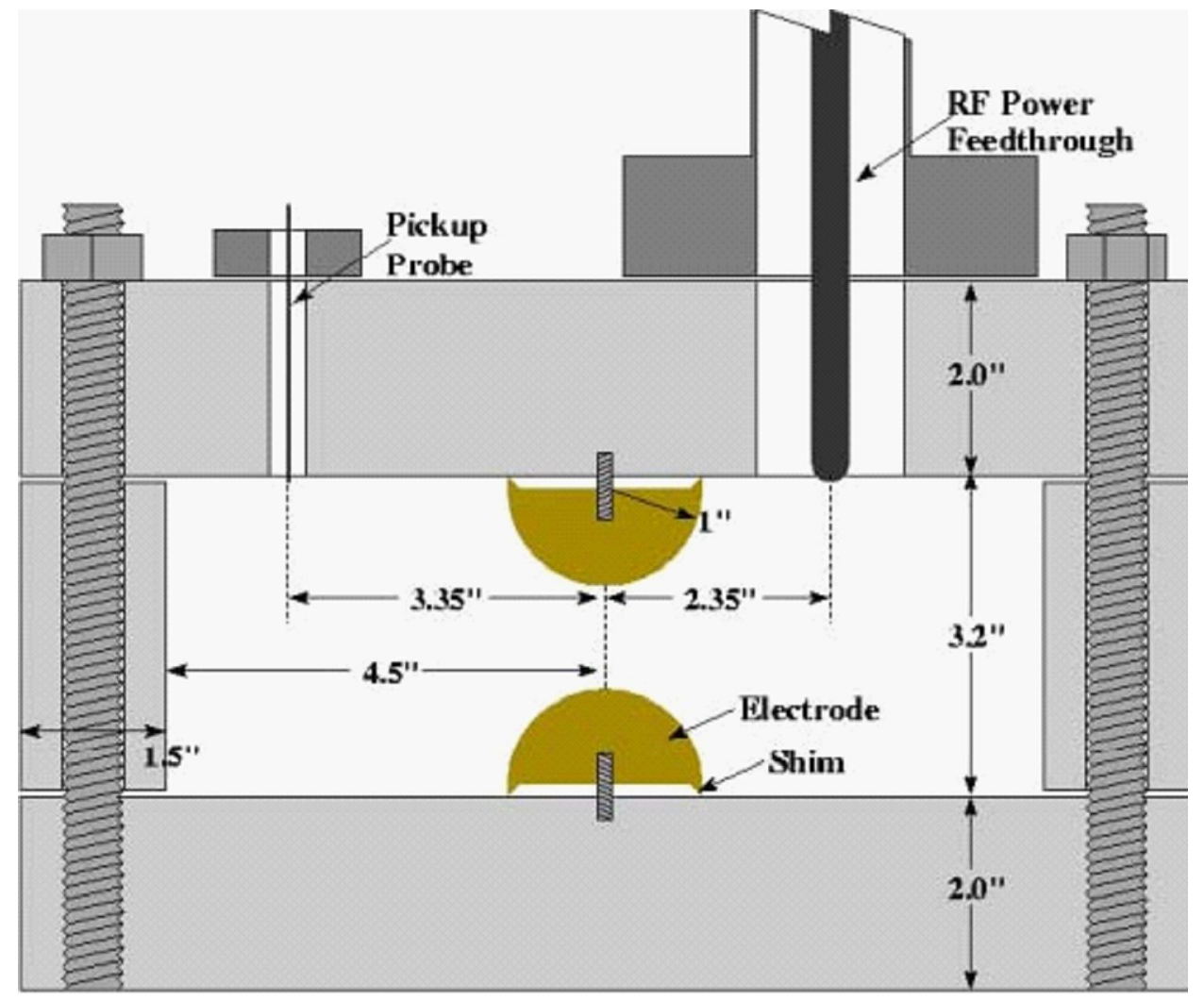

Figure A.1 Drawing of the test cell.

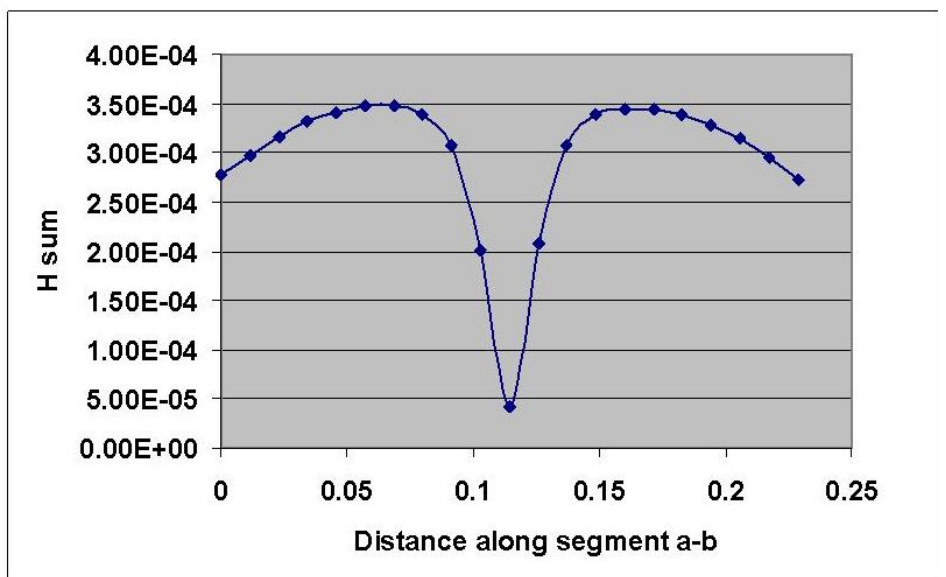

Figure A.2 Cavity magnetic field along mid-plane. The distance is in meters. Ref.[25] 